\title{
Competing for Secrecy in the MISO Interference Channel
}

\author{
S. Ali A. Fakoorian, Member, IEEE, and A. Lee Swindlehurst, Fellow, IEEE
}

\begin{abstract}
A secure communication game is considered for the two-user MISO Gaussian interference channel with confidential messages, where each transmitter aims to maximize the difference between its secrecy rate and the secrecy rate of the other transmitter. In this novel problem, the weaker link tries to minimize the extra secret information obtained by its adversary, while the stronger link attempts to maximize it. We provide an information theoretic formulation for this non-cooperative zero-sum game scenario and determine that, under the assumption of Gaussian signaling at the transmitters, there exists a unique Nash equilibrium solution for the proposed problem. Moreover, we obtain in closed-form the optimal strategies (beamformers) at the transmitters that result in the Nash equilibrium. While the NE strategies are achievable in one shot when full channel state information is available at the transmitters (CSIT), we also propose an iterative step-by-step algorithm that converges to the NE point, but only requires limited CSIT. Numerical results are presented to illustrate the analytical findings and to study the role of different channel parameters on the NE strategies.
\end{abstract}

Index Terms-Game theory, information theory, interference channel, MISO channel, physical-layer security.

\section{INTRODUCTION}

$\mathbf{R}$ ECENT information-theoretic research on secure communication has focused on enhancing security at the physical layer. The wiretap channel, first introduced and studied by Wyner [1], is the most basic physical layer model that captures the problem of communication security. This work led to the development of the notion of perfect secrecy capacity, which quantifies the maximum rate at which a transmitter can reliably send a secret message to its intended recipient, without it being decoded by an eavesdropper. The Gaussian wiretap channel, in which the outputs of the legitimate receiver and the eavesdropper are corrupted by additive white Gaussian noise, was studied in [2]. The secrecy capacity of a Gaussian wiretap channel, which is in general a difficult non-convex optimization problem, has been addressed and solved for in [3]-[7].

Manuscript received December 10, 2011; revised May 06, 2012 and July 26, 2012; accepted October 01, 2012. Date of publication October 09, 2012; date of current version December 12, 2012. The associate editor coordinating the review of this manuscript and approving it for publication was Prof. Merouane Debbah. This work was supported by the U.S. Army Research Office under the Multi-University Research Initiative (MURI) grant W911NF-07-1-0318, and by the National Science Foundation under grant CCF-1117983.

The authors are with the Department of Electrical Engineering and Computer Science, University of California, Irvine, CA 92697-2625 USA (e-mail: afakoori@uci.edu; swindleg@uci.edu).

Color versions of one or more of the figures in this paper are available online at http://ieeexplore.ieee.org.

Digital Object Identifier 10.1109/TSP.2012.2223689
Given the adversarial nature of the wiretap channel and related scenarios with eavesdroppers, many researchers have proposed approaches based on game theory. A physical layer security game is introduced for the MIMO wiretap channel in [8], where the wiretapper possesses the dual capability to act either as a passive eavesdropper or as an active jammer. In [9]-[13], a wiretap channel with an external helper is considered, where the friendly jammer transmits a jamming signal to interfere with the eavesdropper. The problem of finding the optimal jamming power for the helper is formulated via two auction games in [11], assuming the friendly jammer as the auctioneer and the sources as the bidders. Coalition formation games are applied in [12] to improve the physical layer security of wireless nodes through cooperation among the transmitters. In [13], a Stackelburg game is used to investigate the interactions between the transmitter and different friendly jammers, in the presence of an eavesdropper. In [14], zero-sum games are considered for the case that the external jammer helps the eavesdropper by relaying the information signal and/or transmitting interference.

Game theory approaches have also been proposed for the so-called interference channel (IFC). The IFC refers to the case where multiple communication links are simultaneously active in the same time and frequency slot, and hence potentially interfere with or leak information to each other. The most general case for the two-user IFC with confidential messages, where each receiver potentially acts as an eavesdropper for the other link, was studied in [15] where the authors imposed a perfect secrecy constraint for the discrete memoryless interference channel and obtained inner and outer bounds for the perfect secrecy capacity region. They also derived the achievable secrecy rate regions of several simple transmission schemes for single-input single-output (SISO) Gaussian interference channels.

In [16], the Gaussian IFC was considered from a game theoretic perspective with transmitters as selfish players and secrecy rates as utility functions, where each transmitter wishes to maximize its own utility function. An iterative algorithm was proposed to compute the Nash Equilibrium (NE), which corresponds to the operating point where none of the players (transmitters) can improve its situation by unilaterally changing its strategy. Special game setups for the IFC with secrecy constraints are addressed in [17], [18]. In [17], a cognitive radio scenario is considered where only the message from the primary transmitter is considered confidential and must be kept secret at the cognitive receiver. While the primary transmitter and receiver employ a single-user wiretap channel encoder and decoder, respectively, the strategy of the cognitive transmitter is power allocation on the non-secure cognitive message and the noise signal [17]. For this scenario, the NE is established for the 
case that all nodes have a single-antenna (SISO). In [18], a twouser one-sided interference channel with confidential messages is considered, in which one transmitter-receiver pair is interference-free. For this scenario, the NE for the binary deterministic channel is determined. In [19], we investigated an arbitrary two-user multiple-input multiple-output (MIMO) Gaussian interference channel with confidential messages, and studied the achievable secrecy rate region for both non-cooperative and cooperative approaches based on game-theoretic bargaining.

In this paper, we consider a two-user MISO Gaussian interference channel where each multi-antenna transmitter desires to send independent and confidential information to its single-antenna intended recipient, while the other receiver potentially acts as an eavesdropper. For this scenario, we study the case that each transmitter wishes to maximize the difference between its secrecy rate and the secrecy rate of the other link. We show that for this utility function, there exists a unique Nash Equilibrium under the assumption that the transmitters use Gaussian signaling. We also derive a closed-form expression for the optimal strategies (beamforming vectors) at the transmitters that result in the NE solution.

A utility function based on the secrecy rate difference between the two IFC users could arise for example in a tactical military scenario where the two transmitters are adversaries simultaneously sending data from the battlefield back to a command and control center. An approach that only maximized the difference between the standard data rates of the two users would not be appropriate since, even if Link 1 experienced a low data rate, a lack of security in Link 2's message may allow receiver 1 access to high rate information anyway. Furthermore, an approach that simply attempted to maximize each user's secrecy rate wouldn't necessarily provide an advantage over the adversary, who might also be able to achieve a high secrecy rate as well. Instead, we focus on the case where the weaker link tries to minimize the extra secret information obtained by its adversary, while the stronger link attempts to maximize it. In particular, suppose that Link 1 has an advantage over Link 2; in other words, if transmitter $1(\mathrm{Tx} 1)$ acts rationally, it will be able to achieve a higher secrecy rate than $\mathrm{Tx} 2$. By maximizing the secrecy rate difference, Tx 1 maximizes the amount of additional secret information that it is able to send over a given period of time compared with $T \times 2$. On the other hand, Tx2 minimizes the secrecy rate difference in order to reduce the extra secret information that Tx 1 sends. If the IFC scenario is (slowly) time varying, Tx2 could hope that at some future time, the channel conditions will reverse themselves, and it may be able to obtain an advantage and reduce the gap in secret information. If Tx2 had focused solely on maximizing its own secrecy rate without concern for the secrecy of Tx1, the secret information gap it would have to overcome would be much larger. From Tx1's perspective, it wants to maximize the gap as much as possible, so that if the channel conditions become less favorable, there is a wider gap for $\mathrm{Tx} 2$ to overcome.

The remainder of the paper is organized as follows. In Section II, we describe the system model for the Gaussian MISO interference channel and formulate the problem to be solved. In Section III, we determine the existence and uniqueness of the NE and derive the optimal beamformers at the

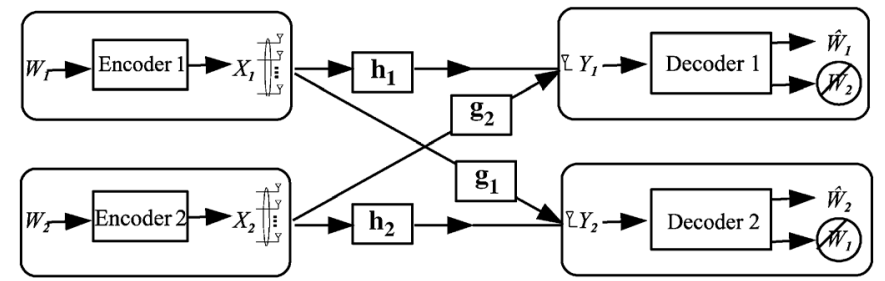

Fig. 1. System model for the two-user MISO interference channel with confidential messages.

transmitters that achieve the NE solution. Numerical results in Section IV are presented to illustrate the proposed solution. Finally, Section V concludes the paper.

Notation: Boldface lowercase letters are used to denote column vectors. Vector-valued random variables are written with non-boldface uppercase letters (e.g., $X$ ), while the corresponding boldface lowercase letter $(\mathbf{x})$ denotes a specific realization of the random variable. Scalar variables are written with non-boldface (lowercase or uppercase) letters. The Hermitian (i.e., conjugate) transpose is denoted by $(.)^{H}$, the matrix trace by $\operatorname{Tr}($.$) , and \mathbf{I}$ indicates an identity matrix. The Euclidean norm of the vector $\mathbf{x}$ is written as $\|\mathbf{x}\|$, mutual information between the random variables $A$ and $B$ is denoted by $I(A ; B)$, and $\mathcal{C N}\left(0, \sigma^{2}\right)$ represents the complex circularly symmetric Gaussian distribution with zero mean and variance $\sigma^{2}$.

\section{System Model AND AsSUMPTIONS}

We consider the two-user Gaussian interference channel shown in Fig. 1, where transmitter 1 with $n_{1}>1$ antennas and transmitter 2 with $n_{2}>1$ antennas intend to send independent and confidential messages $W_{1}$ and $W_{2}$ to single-antenna receivers 1 and 2, respectively. The mathematical model for the signals at the two receivers is given by:

$$
\begin{aligned}
& y_{1}=\mathbf{h}_{1}^{H} \mathbf{x}_{1}+\mathbf{g}_{2}^{H} \mathbf{x}_{2}+z_{1} \\
& y_{2}=\mathbf{h}_{2}^{H} \mathbf{x}_{2}+\mathbf{g}_{1}^{H} \mathbf{x}_{1}+z_{2},
\end{aligned}
$$

where $\mathbf{x}_{i} \in \mathbb{C}^{n_{i} \times 1}$ is the signal vector from transmitter $i, z_{i} \sim$ $\mathcal{C N}\left(0, \sigma_{i}^{2}\right)$ is additive white Gaussian noise at receiver $i$, and the column vectors $\mathbf{h}_{i}$ and $\mathbf{g}_{i}$ represent complex channel gains from transmitter $i$ to its intended receiver and its eavesdropper, respectively. The signal power at transmitter $i$ is constrained by

$$
\operatorname{Tr}\left(E\left\{X_{i} X_{i}^{H}\right\}\right)=\operatorname{Tr}\left(\mathbf{K}_{i}\right) \leq P_{i},
$$

where $\mathbf{K}_{i} \succeq \mathbf{0}$ is the covariance matrix of the $i$ th transmitted signal. We assume that all channels are fixed throughout the entire transmission period, and to begin with, we assume all channels are known to both transmitters. Later, we will consider an approach to deal with the case where limited channel state information is available.

Transmitter $1(\mathrm{Tx} 1)$ attempts to keep its message for receiver 1 (Rx1) confidential from receiver $2(\mathrm{Rx} 2)$, and vice versa. The corresponding information-theoretic secrecy constraint is given by [15]

$$
\lim _{N \rightarrow \infty} \frac{1}{N} I\left(W_{i} ; Y_{j}^{N}\right)=0, \quad i \neq j,
$$

where $N$ is the number of channel uses required for transmitter $i$ to send message $W_{i}$, and $Y_{j}$ is the output at receiver $j$, whose 
realizations for a given set of inputs are given by (1), (2). Theorem 2 of [15] provides the achievable secrecy rates for the IFC with confidential messages:

$$
\begin{aligned}
& R_{1}=\max \left\{0, I\left(V_{1} ; Y_{1}\right)-I\left(V_{1} ; Y_{2} \mid V_{2}\right)\right\} \\
& R_{2}=\max \left\{0, I\left(V_{2} ; Y_{2}\right)-I\left(V_{2} ; Y_{1} \mid V_{1}\right)\right\}
\end{aligned}
$$

over all

$$
\begin{aligned}
& p\left(V_{1}, V_{2}, X_{1}, X_{2}, Y_{1}, Y_{2}\right) \\
& \quad=p\left(V_{1}\right) p\left(V_{2}\right) p\left(X_{1} \mid V_{1}\right) p\left(X_{2} \mid V_{2}\right) p\left(Y_{1}, Y_{2} \mid X_{1}, X_{2}\right),
\end{aligned}
$$

where the pre-coding auxiliary random variables $V_{1}$ and $V_{2}$ (which are independent), represent two independent stochastic encoders.

We study a competitive game where each player (transmitter) aims to maximize the difference between its secrecy rate and the secrecy rate of the other link. We only consider the non-trivial case where both transmitters have positive secrecy rates, i.e., $R_{1}>0$ and $R_{2}>0$. If the strategy chosen by transmitter $i$ leads to $R_{i}=0$, then under our assumed framework, transmitter $i$ would have no reason to transmit information, and would presumably change its role to that of a jammer and only broadcast artificial noise to minimize $R_{j},(i, j \in\{1,2\}, i \neq j)$. Since this case no longer corresponds to an IFC with confidential messages, this will lead to a different game formulation and thus we do not consider this case here. Thus, for Tx 1 the utility function is $R_{1}-R_{2}$, which using (5), (6) is given by

$$
R_{1}-R_{2}=I\left(V_{1}, V_{2} ; Y_{1}\right)-I\left(V_{1}, V_{2} ; Y_{2}\right) .
$$

While Tx1 chooses a strategy that maximizes (7), Tx2 tries to minimize it, or equivalently maximize its own utility function $R_{2}-R_{1}$. Since the sum of the utility functions for the two players is zero, this non-cooperative competitive game is a zero-sum game [20]. Thus, at the Nash Equilibrium, where no player can improve its utility by unilaterally changing its strategy, we must have:

$$
\begin{aligned}
\left(V_{1}^{*}, V_{2}^{*}\right) & =\arg \max _{V_{1}} \min _{V_{2}} I\left(V_{1}, V_{2} ; Y_{1}\right)-I\left(V_{1}, V_{2} ; Y_{2}\right) \\
& =\arg \max _{V_{2}} \min _{V_{1}} I\left(V_{1}, V_{2} ; Y_{2}\right)-I\left(V_{1}, V_{2} ; Y_{1}\right),
\end{aligned}
$$

where $V_{i}^{*}$ represents the optimal pre-coding random variable at transmitter $i$ for the NE solution.

Eqs. (8), (9) state that if this game has an equilibrium solution, the max-min and min-max strategies will be the same, and both will yield the strategy profile $\left(V_{1}^{*}, V_{2}^{*}\right)$. In other words, the NE strategy for player (transmitter) $i$ corresponds to the best move for Tx $i$ when Tx $i$ must move first and choose its strategy only once, assuming that Tx $j$ plays next, knows Tx $i$ 's strategy and chooses a strategy that minimizes Tx $i$ 's utility function [21]. To solve (8), (9) in the most general case, one would need to determine the optimal signaling distribution for $X_{i}$, as well as th optimal choice for prefix random variables $V_{i}$. While these are still open problems for the IFC, the following lemma establishes that under the assumption of Gaussian signaling, prefix coding at the transmitters is not required, a result that will allow us to obtain a direct solution for the NE.
Lemma 1: Under the assumption of Gaussian signaling at the transmitters, a Gaussian $V_{i}=X_{i}$ is an optimal solution at transmitter $i$ to achieve the NE point in (8), (9).

Proof: We will show the optimality of $V_{1}=X_{1}$. Writing the mutual information in terms of entropy, i.e., $I(U ; Y)=$ $h(Y)-h(Y \mid U)$, from (8) we have

$$
\begin{gathered}
\arg \max _{V_{1}} I\left(V_{1}, V_{2} ; Y_{1}\right)-I\left(V_{1}, V_{2} ; Y_{2}\right) \\
=\arg \max _{V_{1}}\left\{h\left(Y_{1}\right)-h\left(Z_{1}\right)-h\left(Y_{2}\right)+h\left(Z_{2}\right)\right\} \\
=\arg \max _{V_{1}}\left\{h\left(Y_{1}\right)-h\left(\mathbf{g}_{2}^{H} X_{2}+Z_{1}\right)-h\left(Y_{2}\right)\right. \\
\left.\quad+h\left(\mathbf{h}_{2}^{H} X_{2}+Z_{2}\right)\right\} \\
=\arg \max _{V_{1}}\left\{I\left(V_{1} ; Y_{1}\right)-I\left(V_{1} ; Y_{2}\right)\right\} .
\end{gathered}
$$

Since $X_{2}$ is Gaussian, (10) represents the single-letter expression for the secrecy capacity of a Gaussian wiretap channel [6]. It has been shown, for example in [3], that for the Gaussian wiretap channel, a Gaussian $V_{1}=X_{1}$ yields an optimal solution. A description similar to that above would also apply to Tx2 for the optimality of a Gaussian $V_{2}=X_{2}$ at the NE point.

Lemma 1 indicates that to achieve the NE point for the proposed Gaussian IFC, the transmitters do not need to apply prefix coding. Thus a matrix characterization of the optimization problem (8), (9) can be given as follows (where as mentioned above we have assumed that both users achieve positive non-zero secrecy rates):

$$
\begin{aligned}
\left(\mathbf{K}_{1}^{*}, \mathbf{K}_{2}^{*}\right)= & \arg \max _{\mathbf{K}_{1} \succeq 0} \min _{\mathbf{K}_{2} \succeq 0} \log \left(1+\mathbf{h}_{1}^{H} \mathbf{K}_{1} \mathbf{h}_{1}+\mathbf{g}_{2}^{H} \mathbf{K}_{2} \mathbf{g}_{2}\right) \\
& -\log \left(1+\mathbf{h}_{2}^{H} \mathbf{K}_{2} \mathbf{h}_{2}+\mathbf{g}_{1}^{H} \mathbf{K}_{1} \mathbf{g}_{1}\right) \\
= & \arg \min _{\mathbf{K}_{2} \succeq 0} \max _{1} \log \left(1+\mathbf{h}_{1}^{H} \mathbf{K}_{1} \mathbf{h}_{1}+\mathbf{g}_{2}^{H} \mathbf{K}_{2} \mathbf{g}_{2}\right) \\
& -\log \left(1+\mathbf{h}_{2}^{H} \mathbf{K}_{2} \mathbf{h}_{2}+\mathbf{g}_{1}^{H} \mathbf{K}_{1} \mathbf{g}_{1}\right),
\end{aligned}
$$

where the non-convex optimization problems are solved under the power constraint given in (3), and we have assumed without loss of generality that $\sigma_{1}^{2}=\sigma_{2}^{2}=1$.

Consider for example Tx1 in problem (11). One can easily confirm that for a given $\mathbf{K}_{2}$, the optimum $\mathbf{K}_{1}$ that maximizes (11) also solves the following problem

$\max _{\mathbf{K}_{1} \succeq 0} \log \left(1+\frac{\mathbf{h}_{1}^{H} \mathbf{K}_{1} \mathbf{h}_{1}}{1+\mathbf{g}_{2}^{H} \mathbf{K}_{2} \mathbf{g}_{2}}\right)-\log \left(1+\frac{\mathbf{g}_{1}^{H} \mathbf{K}_{1} \mathbf{g}_{1}}{1+\mathbf{h}_{2}^{H} \mathbf{K}_{2} \mathbf{h}_{2}}\right)$.

The problem in (13) is equivalent to a simple MISO Gaussian wiretap channel, where the equivalent "noise" at the receiver and the eavesdropper is Gaussian with variances $\left(1+\mathbf{g}_{2}^{H} \mathbf{K}_{2} \mathbf{g}_{2}\right)$ and (1+ $\left.\mathbf{h}_{2}^{H} \mathbf{K}_{2} \mathbf{h}_{2}\right)$, respectively. It is proved in [4] and [22, Theorem 3] that for the standard MISO Gaussian wiretap channel, the optimal $\mathbf{K}_{1}$ that achieves the secrecy capacity is rank-one. More precisely, Gaussian signaling in the form of beamforming is optimal [4]. Thus, to achieve the Nash equilibria for the interference channel, the transmit signal at Tx 1 must be represented as $\mathbf{x}_{1}=\mathbf{w}_{1} s_{1}$ where $s_{1}$ is a Gaussian encoded information symbol broadcast by Tx1 using the beamforming vector $\mathbf{w}_{1}$. A description similar to that above would also apply to Tx2, i.e., $\mathbf{x}_{2}=\mathbf{w}_{2} s_{2}$. Without loss of generality, 
we normalize $\mathbf{w}_{i}$ to have unit length, $\mathbf{w}_{i}^{H} \mathbf{w}_{i}=1$, so that $E\left(\left|s_{i}\right|^{2}\right) \leq P_{i}$ for $i=1,2$.

Using the above facts, the optimization problem in (11), (12) can be rewritten as

$$
\begin{aligned}
\left(\mathbf{w}_{1}^{*}, \mathbf{w}_{2}^{*}\right) & =\arg \max _{\left\|\mathbf{w}_{1}\right\|=1\left\|\mathbf{w i n}_{2}\right\|=1} \mathcal{F} \\
& =\arg \min _{\left\|\mathbf{w}_{2}\right\|=1\left\|\mathbf{w a x}_{1}\right\|=1} \mathcal{F},
\end{aligned}
$$

where $\mathcal{F}$ is given by

$$
\begin{aligned}
\mathcal{F}= & R 1-R 2 \\
= & \log \left(1+P_{1} \mathbf{h}_{1}^{H} \mathbf{w}_{1} \mathbf{w}_{1}^{H} \mathbf{h}_{1}+P_{2} \mathbf{g}_{2}^{H} \mathbf{w}_{2} \mathbf{w}_{2}^{H} \mathbf{g}_{2}\right) \\
& -\log \left(1+P_{2} \mathbf{h}_{2}^{H} \mathbf{w}_{2} \mathbf{w}_{2}^{H} \mathbf{h}_{2}+P_{1} \mathbf{g}_{1}^{H} \mathbf{w}_{1} \mathbf{w}_{1}^{H} \mathbf{g}_{1}\right) .
\end{aligned}
$$

Note that in problems (14), (15) we have used the fact that to achieve the NE solution, each transmitter must transmit at its maximum power. This results from the observation that, to achieve the secrecy capacity of the equivalent wiretap channel (given for example by (13) for Tx1), the transmitter uses its maximum power, as shown in [22, App. A]. From now on, for notational simplicity we omit the scalar $P_{i}$ by absorbing it into the channel vector:

$$
\begin{aligned}
\sqrt{P_{i}} \mathbf{h}_{i} & \rightarrow \mathbf{h}_{i} \\
\sqrt{P_{i}} \mathbf{g}_{i} & \rightarrow \mathbf{g}_{i} .
\end{aligned}
$$

Thus, $\mathcal{F}$ can be rewritten as

$$
\begin{aligned}
\mathcal{F}=\log \left(1+\mathbf{h}_{1}^{H} \mathbf{w}_{1} \mathbf{w}_{1}^{H} \mathbf{h}_{1}+\mathbf{g}_{2}^{H} \mathbf{w}_{2} \mathbf{w}_{2}^{H} \mathbf{g}_{2}\right) & \\
& -\log \left(1+\mathbf{h}_{2}^{H} \mathbf{w}_{2} \mathbf{w}_{2}^{H} \mathbf{h}_{2}+\mathbf{g}_{1}^{H} \mathbf{w}_{1} \mathbf{w}_{1}^{H} \mathbf{g}_{1}\right) .
\end{aligned}
$$

\section{NASH EQUILIBRIUM STRATEGY}

For the MISO Gaussian interference channel in which we are interested, each transmitter aims to maximize the difference between its secrecy rate and the secrecy rate of the other link. In order to show that there exists a Nash equilibrium for this scenario, we need to show that there exists a pair of beamforming vectors $\left(\mathbf{w}_{1}^{*}, \mathbf{w}_{2}^{*}\right)$ for which problems (14) and (15) are simultaneously satisfied. It should be noted that since $\mathcal{F}$ is a non-convex function of $\mathbf{w}_{1}$ and $\mathbf{w}_{2}$, problems (14) and (15) are different.

\section{A. Critical Points of $\mathcal{F}$}

Referring to problems (14) and (15), we have:

Lemma 2: Any $\mathbf{w}_{1}$ that maximizes $\mathcal{F}$ for a given $\mathbf{w}_{2}$, and any $\mathbf{w}_{2}$ which minimizes $\mathcal{F}$ for a given $\mathbf{w}_{1}$, must have the following form ${ }^{1}$ :

$$
\mathbf{w}_{i}=\cos \left(\theta_{i}\right) \frac{\mathbf{P}_{g_{i}} \mathbf{h}_{i}}{\left\|\mathbf{P}_{g_{i}} \mathbf{h}_{i}\right\|}+\sin \left(\theta_{i}\right) \frac{\mathbf{P}_{g_{i}}^{\perp} \mathbf{h}_{i}}{\left\|\mathbf{P}_{g_{i}}^{\perp} \mathbf{h}_{i}\right\|} \quad 0 \leq \theta_{i} \leq \frac{\pi}{2}
$$

where $\mathbf{P}_{g_{i}}=\left(\mathbf{g}_{i}^{H} \mathbf{g}_{i}\right)^{-1} \mathbf{g}_{i} \mathbf{g}_{i}^{H}$ denotes the orthogonal projection onto span $\left(\mathbf{g}_{i}\right)$ and $\mathbf{P}_{g_{i}}^{\perp}=\mathbf{I}-\mathbf{P}_{g_{i}}$ denotes the projection onto its orthogonal complement.

Proof: The proof follows exactly the same steps as Corollary 1 in [23].

Let $\beta_{i}$ denote the angle between $\mathbf{h}_{i}, \mathbf{g}_{i}$, which is given by

$$
\beta_{i}=\cos ^{-1}\left(\frac{\left\|\mathbf{P}_{g_{i}} \mathbf{h}_{i}\right\|}{\left\|\mathbf{h}_{i}\right\|}\right) \text {. }
$$

${ }^{1}$ Clearly, for any real-valued $\gamma, e^{j \gamma} \mathbf{w}_{i}$ would also be acceptable.
Using (20), we have:

$$
\begin{aligned}
\mathbf{g}_{i}^{H} \mathbf{w}_{i} \mathbf{w}_{i}^{H} \mathbf{g}_{i} & =\cos ^{2}\left(\theta_{i}\right) \mathbf{g}_{i}^{H} \mathbf{g}_{i} \\
\mathbf{h}_{i}^{H} \mathbf{w}_{i} \mathbf{w}_{i}^{H} \mathbf{h}_{i} & =\cos ^{2}\left(\theta_{i}-\beta_{i}\right) \mathbf{h}_{i}^{H} \mathbf{h}_{i} .
\end{aligned}
$$

Define

$$
\begin{aligned}
h_{i} & =\mathbf{h}_{i}^{H} \mathbf{h}_{i}=\left\|\mathbf{h}_{i}\right\|^{2} \\
g_{i} & =\mathbf{g}_{i}^{H} \mathbf{g}_{i}=\left\|\mathbf{g}_{i}\right\|^{2},
\end{aligned}
$$

and then use (22)-(25) in (19) to rewrite $\mathcal{F}$ as

$$
\begin{aligned}
\mathcal{F}=\log \left(1+h_{1} \cos ^{2}\left(\theta_{1}-\beta_{1}\right)+g_{2} \cos ^{2}\left(\theta_{2}\right)\right) & \\
& -\log \left(1+h_{2} \cos ^{2}\left(\theta_{2}-\beta_{2}\right)+g_{1} \cos ^{2}\left(\theta_{1}\right)\right) .
\end{aligned}
$$

Thus, based on Lemma 2, the optimization problems (14), (15) can be reparameterized in terms of simply the angles $\theta_{1}, \theta_{2}$ :

$$
\begin{aligned}
\left(\theta_{1}^{*}, \theta_{2}^{*}\right) & =\arg \max _{0 \leq \theta_{1} \leq \frac{\pi}{2}} \min _{0 \leq \theta_{2} \leq \frac{\pi}{2}} \mathcal{F} \\
& =\arg \min _{0 \leq \theta_{2} \leq \frac{\pi}{2}} \max _{0 \leq \theta_{1} \leq \frac{\pi}{2}} \mathcal{F} .
\end{aligned}
$$

To obtain the Nash equilibria, we must find a pair of angles $\left(\theta_{1}^{*}, \theta_{2}^{*}\right) \in\left\{\left[0, \frac{\pi}{2}\right] \times\left[0, \frac{\pi}{2}\right]\right\}$ for which problems (27), (28) are simultaneously satisfied. Our approach to finding the NE is as follows: we first find the critical point of $\mathcal{F}$, namely $\left(\bar{\theta}_{1}, \bar{\theta}_{2}\right)$, where the first-order partial derivatives are equal to zero. Then we show that $\left(\bar{\theta}_{1}, \bar{\theta}_{2}\right)=\left(\theta_{1}^{*}, \theta_{2}^{*}\right)$, where the pair $\left(\theta_{1}^{*}, \theta_{2}^{*}\right)$ is the concurrent solution of (27), (28), and gives the optimal beamformers for the transmitters at the NE point.

Theorem 1: The unique critical point of $\mathcal{F}$ in the range of $\left\{\left[0, \frac{\pi}{2}\right] \times\left[0, \frac{\pi}{2}\right]\right\}$ is given

$\bar{\theta}_{1}=\tan ^{-1}\left(\frac{\sqrt{1-2 \cos \left(2 \beta_{1}\right) \frac{g_{1}}{h_{1}} m+\frac{g_{1}^{2}}{h_{1}^{2}} m^{2}}-\cos \left(2 \beta_{1}\right)+\frac{g_{1}}{h_{1}} m}{\sin \left(2 \beta_{1}\right)}\right)$

$\bar{\theta}_{2}=\tan ^{-1}\left(\frac{\sqrt{1-2 \cos \left(2 \beta_{2}\right) \frac{g_{2}}{h_{2}} \frac{1}{m}+\frac{g_{2}^{2}}{h_{2}^{2}} \frac{1}{m^{2}}}-\cos \left(2 \beta_{2}\right)+\frac{g_{2}}{h_{2}} \frac{1}{m}}{\sin \left(2 \beta_{2}\right)}\right)$

where $m$ is the only real, positive solution to the equation

$$
\begin{gathered}
1+\frac{g_{2}}{2}+\frac{h_{1}}{2}\left(1+\sqrt{1-2 \cos \left(2 \beta_{1}\right) \frac{g_{1}}{h_{1}} m+\frac{g_{1}^{2}}{h_{1}^{2}} m^{2}}\right)=m \\
+m \frac{g_{1}}{2}+m \frac{h_{2}}{2}\left(1+\sqrt{1-2 \cos \left(2 \beta_{2}\right) \frac{g_{2}}{h_{2}} \frac{1}{m}+\frac{g_{2}^{2}}{h_{2}^{2}} \frac{1}{m^{2}}}\right)
\end{gathered}
$$

and can be found as one of the roots to the fourth-order polynomial

$$
a m^{4}+b m^{3}+c m^{2}+d m+e=0,
$$

where the coefficients $a-e$ are defined in Appendix A.

Proof: Please refer to Appendix A. 


\section{B. Optimality of the Critical Point in Achieving the Nash Equilibria}

Theorem 1 obtains the only critical point of $\mathcal{F}$ in the range of interest $\left(\theta_{1}, \theta_{2}\right) \in\left\{\left[0, \frac{\pi}{2}\right] \times\left[0, \frac{\pi}{2}\right]\right\}$. In this subsection, we show that the obtained critical point is indeed the NE point. Mathematically, we want to show that $\left(\bar{\theta}_{1}, \bar{\theta}_{2}\right)=\left(\theta_{1}^{*}, \theta_{2}^{*}\right)$; i.e., $\left(\bar{\theta}_{1}, \bar{\theta}_{2}\right)$ gives the NE point by simultaneously solving $(27),(28)$. To prove this, we first show the following:

$$
\begin{aligned}
& \bar{\theta}_{1}=\arg \max _{\theta_{1}} \mathcal{F}\left(\theta_{1}, \bar{\theta}_{2}\right) \\
& \bar{\theta}_{2}=\arg \min _{\theta_{2}} \mathcal{F}\left(\bar{\theta}_{1}, \theta_{2}\right) .
\end{aligned}
$$

Theorem 2: The critical point $\left(\bar{\theta}_{1}, \bar{\theta}_{2}\right)$ of $\mathcal{F}$ in the range of $\left\{\left[0, \frac{\pi}{2}\right] \times\left[0, \frac{\pi}{2}\right]\right\}$ satisfies (33), (34).

Proof: Please refer to Appendix B.

So far, by proving (33), (34), we have shown that if Tx1 chooses $\bar{\theta}_{1}$ as its strategy, then the best strategy from the viewpoint of Tx2 will be $\bar{\theta}_{2}$, and vice versa. Now let us return to the main problem of this subsection. We want to show that the unique critical point of $\mathcal{F}$ in the range of interest is in fact the NE point, i.e., $\left(\bar{\theta}_{1}, \bar{\theta}_{2}\right)=\left(\theta_{1}^{*}, \theta_{2}^{*}\right)$.

Theorem 3: The critical point $\left(\bar{\theta}_{1}, \bar{\theta}_{2}\right)$ of $\mathcal{F}$ in the range of $\left\{\left[0, \frac{\pi}{2}\right] \times\left[0, \frac{\pi}{2}\right]\right\}$ is the Nash equilibrium which simultaneously satisfies (27), (28).

Proof: We have:

$$
\begin{aligned}
\min _{\theta_{2}} \max _{\theta_{1}} \mathcal{F}\left(\theta_{1}, \theta_{2}\right) & \leq \max _{\theta_{1}} \mathcal{F}\left(\theta_{1}, \bar{\theta}_{2}\right) \\
& =\mathcal{F}\left(\bar{\theta}_{1}, \bar{\theta}_{2}\right)=\min _{\theta_{2}} \mathcal{F}\left(\bar{\theta}_{1}, \theta_{2}\right) \\
& \leq \max _{\theta_{1}} \min _{\theta_{2}} \mathcal{F}\left(\theta_{1}, \theta_{2}\right)
\end{aligned}
$$

where the equalities in (35) come from Theorem 2. One can show that for any function $\mathcal{F}$

$$
\max _{\theta_{1}} \min _{\theta_{2}} \mathcal{F}\left(\theta_{1}, \theta_{2}\right) \leq \min _{\theta_{2}} \max _{\theta_{1}} \mathcal{F}\left(\theta_{1}, \theta_{2}\right) .
$$

From (36) and (37) we have

$$
\max _{\theta_{1}} \min _{\theta_{2}} \mathcal{F}\left(\theta_{1}, \theta_{2}\right)=\min _{\theta_{2}} \max _{\theta_{1}} \mathcal{F}\left(\theta_{1}, \theta_{2}\right)=\mathcal{F}\left(\bar{\theta}_{1}, \bar{\theta}_{2}\right)
$$

which gives the final result $\left(\theta_{1}^{*}, \theta_{2}^{*}\right)=\left(\bar{\theta}_{1}, \bar{\theta}_{2}\right)$ and completes the proof.

\section{Discussion}

Using Theorem 3, we have closed form expressions for the optimal strategies at the transmitters for the NE as follows

$\theta_{1}^{*}=\tan ^{-1}\left(\frac{\sqrt{1-2 \cos \left(2 \beta_{1}\right) \frac{g_{1}}{h_{1}} m+\frac{g_{1}^{2}}{h_{1}^{2}} m^{2}}-\cos \left(2 \beta_{1}\right)+\frac{g_{1}}{h_{1}} m}{\sin \left(2 \beta_{1}\right)}\right)$

$\theta_{2}^{*}=\tan ^{-1}\left(\frac{\sqrt{1-2 \cos \left(2 \beta_{2}\right) \frac{g_{2}}{h_{2}} \frac{1}{m}+\frac{g_{2}^{2}}{h_{2}^{2}} \frac{1}{m^{2}}}-\cos \left(2 \beta_{2}\right)+\frac{g_{2}}{h_{2}} \frac{1}{m}}{\sin \left(2 \beta_{2}\right)}\right)$ where $m$ is found as explained in Theorem 1 . Note that the corresponding optimal beamformer at transmitter $i, \mathbf{w}_{i}^{*}$, is obtained by replacing $\theta_{i}^{*}$ in the RHS of (20).

Referring to (39) and (40), we have the following observations about the NE strategies $\left(\theta_{1}^{*}, \theta_{2}^{*}\right)$ :

1) Since the utility function $\mathcal{F}$ has only one critical point in the range of $\left(\theta_{1}, \theta_{2}\right) \in\left\{\left[0, \frac{\pi}{2}\right] \times\left[0, \frac{\pi}{2}\right]\right\}$, and since any NE point is a critical point by the KKT conditions, the NE strategies for each user, given by (39), (40), are unique.

2) The optimal NE strategy for either transmitter is a function of $\beta_{i}, h_{i}$, and $g_{i}$ for $i=1,2$. The quantities $h_{i}$ and $g_{i}$ only depend on the average transmit power $P_{i}$ and the norms of the channel vectors $\mathbf{h}_{i}$ and $\mathbf{g}_{i}$, as shown in (17), (18) and (24), (25). Thus, any set of channel vectors for which $h_{i}, g_{i}$ and $\beta_{i}$ remain unchanged will lead to the same NE solution $\left(\theta_{1}^{*}, \theta_{2}^{*}\right)$. For example, the two sets of channel vectors shown in Fig. 2 result in the same $\left(\theta_{1}^{*}, \theta_{2}^{*}\right)$ solution. While $\left(\theta_{1}^{*}, \theta_{2}^{*}\right)$ is unchanged in such cases, the actual beamforming vectors $\mathbf{w}_{1}^{*}$ and $\mathbf{w}_{2}^{*}$ will be different.

3) Consider, for example, Tx1. From (39), one can easily confirm that there are two cases for which the optimal NE strategy at $\mathrm{Tx} 1$, defined by $\theta_{1}^{*}$, is completely independent of the system parameters at $\operatorname{Tx} 2\left(P_{2}, \mathbf{h}_{2}, \mathbf{g}_{2}\right)$. These special cases are $\beta_{1}=0$ and $\beta_{1}=\frac{\pi}{2}$, which result in $\theta_{1}^{*}=0$ and $\theta_{1}^{*}=\frac{\pi}{2}$, respectively. In this situation, the corresponding optimal NE beamformer at Tx $1, \mathbf{w}_{1}^{*}$, is in the direction of $\mathbf{h}_{1}$, either orthogonal to $\mathbf{g}_{1}$ (for $\beta_{1}=\frac{\pi}{2}$ ) or parallel to it (for $\beta_{1}=0$ ).

4) Finally, from (5), (6), one can easily confirm that the secrecy rate of transmitter $i$ at the Nash equilibrium point is given by

$$
\begin{aligned}
R_{i}= & \log \left(1+\frac{P_{i} \mathbf{h}_{i}^{H} \mathbf{w}_{i}^{*} \mathbf{w}_{i}^{* H} \mathbf{h}_{i}}{1+P_{j} \mathbf{g}_{j}^{H} \mathbf{w}_{j}^{*} \mathbf{w}_{j}^{* H} \mathbf{g}_{j}}\right) \\
& -\log \left(1+P_{i} \mathbf{g}_{i}^{H} \mathbf{w}_{i}^{*} \mathbf{w}_{i}^{* H} \mathbf{g}_{i}\right) \\
= & \log \left(1+\frac{P_{i} \mathbf{h}_{i}^{H} \mathbf{h}_{i} \cos ^{2}\left(\theta_{i}^{*}-\beta_{i}\right)}{1+P_{j} \mathbf{g}_{j}^{H} \mathbf{g}_{j} \cos ^{2}\left(\theta_{j}^{*}\right)}\right) \\
& -\log \left(1+P_{i} \mathbf{g}_{i}^{H} \mathbf{g}_{i} \cos ^{2}\left(\theta_{i}^{*}\right)\right) .
\end{aligned}
$$

\section{Iterative Algorithm}

In the above sections, we have fully characterized the NE point in closed-form assuming both transmitters have full channel state information (CSI), or in other words knowledge of $\mathbf{g}_{i}, \mathbf{h}_{i}$ for $i=1,2$. Under this assumption, the transmitters can both use Theorem 1 to calculate their respective transmit beamformer $\mathbf{w}_{i}$, and the NE operating point is achieved in one shot. In the discussion that follows, we discuss how the NE can still be achieved in situations where only limited CSI (defined below) is available to each transmitter. A "best response" iterative algorithm is presented where each transmitter alternately chooses its beamformer to maximize the secrecy rate difference given the adversary's current choice of its own transmit beamformer. As we showed earlier using (11)-(13), this problem is equivalent to maximizing the secrecy rate of a certain MISO Gaussian wiretap channel, and the solution can 


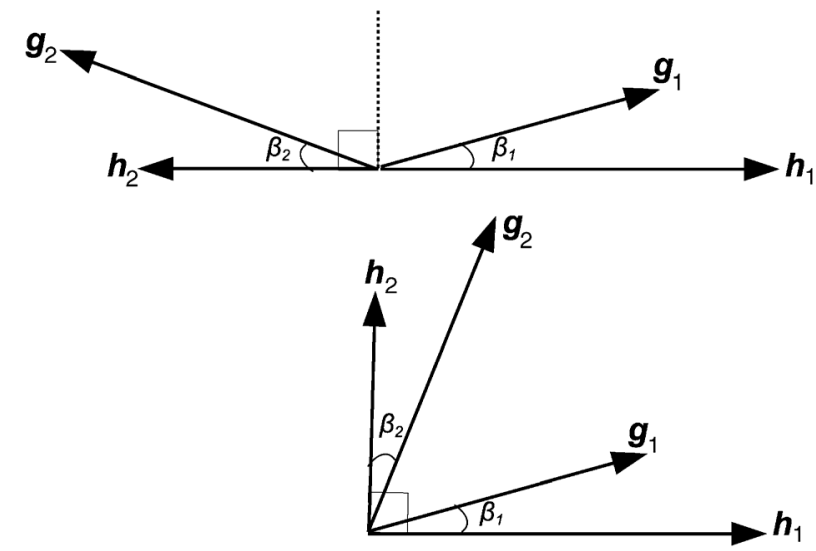

Fig. 2. Examples of two different channel realizations that yield the same NE strategies.

be obtained in closed-form using, for example, the approach of [4]. Although full CSIT is not available, we will assume (see below) that sufficient CSIT is at hand for solving this equivalent wiretap problem. The algorithm is presented below assuming without loss of generality that Tx1 updates its transmit strategy $\left(\theta_{1}\right.$ or equivalently $\left.\mathbf{w}_{1}\right)$ first.

1) Set $l=1$. Initialize $\mathbf{K}_{1}^{(0)}$ and $\mathbf{K}_{2}^{(0)}$ (for example $\mathbf{K}_{i}^{(0)}=$ $P_{i} \frac{\mathbf{h}_{i} \mathbf{h}_{i}^{H}}{\left\|\mathbf{h}_{i}\right\|^{2}}$, i.e. $\mathbf{w}_{i}=\frac{\mathbf{h}_{i}}{\left\|\mathbf{h}_{i}\right\|}$ or $\left.\theta_{i}=\beta_{i}\right)$

2) Tx1 chooses its "best response" by maximizing its utility function, which is equivalent to (13). In order to solve (13), Tx1 requires knowledge of vectors $\mathbf{h}_{1}$ and $\mathbf{g}_{1}$ and scalars $\mathbf{g}_{2}^{H} \mathbf{K}_{2}^{(l-1)} \mathbf{g}_{2}$ and $\mathbf{h}_{2}^{H} \mathbf{K}_{2}^{(l-1)} \mathbf{h}_{2}$. We assume that Rx1 feeds back $\mathbf{h}_{1}$ and its current SINR

$$
\mathrm{SINR}_{1}=\frac{\mathbf{h}_{1}^{H} \mathbf{K}_{1}^{(l-1)} \mathbf{h}_{1}}{1+\mathbf{g}_{2}^{H} \mathbf{K}_{2}^{(l-1)} \mathbf{g}_{2}}
$$

to $\mathrm{Tx} 1$, and that $\mathrm{Rx} 2$ feeds back $\mathbf{h}_{2}$ and $\mathrm{SINR}_{2}$ to $\mathrm{Tx} 2$. From Rx1's transmission, $\mathrm{Tx} 1$ can obtain $\mathbf{h}_{1}$ and $\mathbf{g}_{2}^{H} \mathbf{K}_{2}^{(l-1)} \mathbf{g}_{2}$. By listening to Rx2's transmissions, Tx1 can obtain $\mathbf{g}_{1}$ and it can also deduce the scalar $\mathbf{h}_{2}^{H} \mathbf{K}_{2}^{(l-1)} \mathbf{h}_{2}$ by intercepting Rx2's SINR feedback, since with knowledge of $g_{1}$, Tx1 is aware of the interference it produces at Rx2. Since Tx1 only needs to solve (13) at this stage, it does not require explicit knowledge of $\mathbf{h}_{2}$ and $\mathbf{g}_{2}$, and thus the assumption of full CSI is relaxed. Note that the $\theta_{1}$ corresponding to the optimal solution of (13) is uniquely characterized using an argument similar to that made in Appendix B in obtaining (63).

3) $\mathbf{K}_{2}^{(l)}$ is updated by repeating the previous step for $\mathrm{Tx} 2$ given the covariance $\mathbf{K}_{1}^{(l)}\left(=P_{1} \mathbf{w}_{1} \mathbf{w}_{1}^{H}\right)$ computed by $\mathrm{Tx} 1$ in the previous step.

4) Increment $l \rightarrow l+1$. Repeat steps 2 and 3 until both transmitters perceive a small enough difference in the transmit parameters of their opponent:

$$
\sum_{i=1}^{2}\left|\mathbf{h}_{i}^{H}\left(\mathbf{K}_{i}^{(l)}-\mathbf{K}_{i}^{(l-1)}\right) \mathbf{h}_{i}\right|+\sum_{i=1}^{2}\left|\mathbf{g}_{i}^{H}\left(\mathbf{K}_{i}^{(l)}-\mathbf{K}_{i}^{(l-1)}\right) \mathbf{g}_{i}\right|<\epsilon .
$$

Convergence is guaranteed for the above algorithm based on a well-known result for the two-player zero-sum game, where if

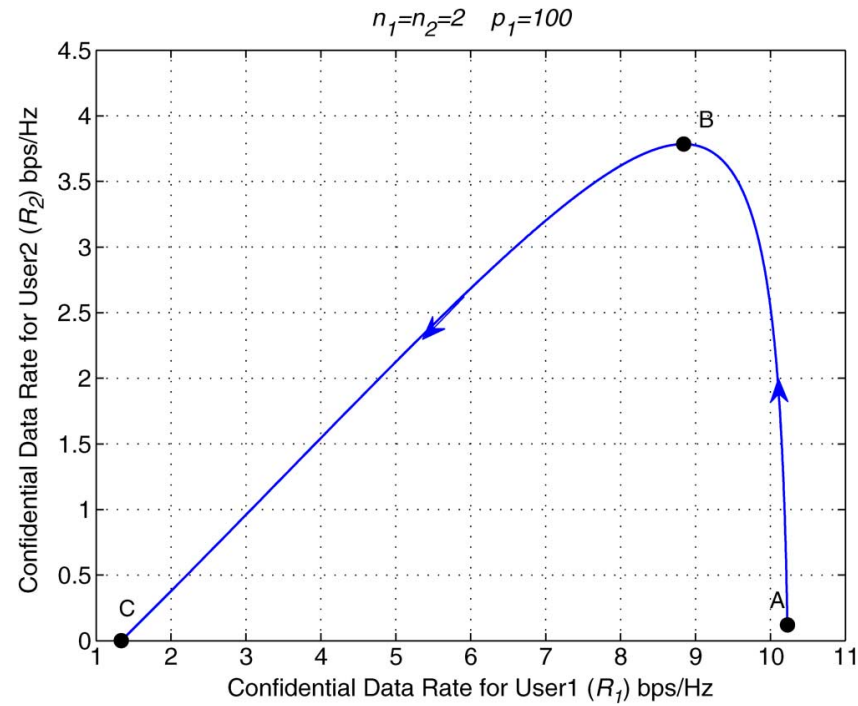

Fig. 3. Track of NE points in response to the changes of $P_{2}$ from 0.01 to 58.75. $\mathbf{h}_{1}=\left[\begin{array}{ll}4 & 0\end{array}\right]^{T}, \mathbf{g}_{1}=[\sqrt{33}]^{T}, \mathbf{h}_{2}=\left[\begin{array}{ll}0 & 3\end{array}\right]^{T}, \mathbf{g}_{2}=\frac{1}{2}\left[\begin{array}{ll}1 & 1\end{array}\right]^{T}$.

each player keeps playing its "best response," then the process will ultimately converge to the NE point [26]. More precisely, similar to the argument made in [16], since the best response for each player is unique in its strategy space, and since the strategy space for each player is closed and bounded, the algorithm converges to the unique NE point, whose existence we have already established. As we will illustrate in the next section, convergence is typically achieved in a small number of iterations, even when the number of antennas at the transmitters is large. Note that the best response for each transmitter is chosen in steps I and II by assuming an IFC scenario, even though prior to convergence the transmitters are not transmitting simultaneously and technically an IFC is not present. For example, Tx1 solves (13) for its best response, where (13) comes from (11) which is the utility function for Tx1 under an IFC system model. This is not a problem since ultimately, upon convergence, both transmitters will be active at the same time and an IFC results.

\section{NumericAl Results}

In this section, we present numerical results to illustrate our theoretical findings. For the first example, we assume $n_{1}=$ $n_{2}=2, P_{1}=100$. The channel vectors $\operatorname{are}^{2} \mathbf{h}_{1}=\left[\begin{array}{ll}4 & 0\end{array}\right]^{T}$, $\mathbf{g}_{1}=\left[\begin{array}{ll}\sqrt{3} & 3\end{array}\right]^{T}, \mathbf{h}_{2}=\left[\begin{array}{ll}0 & 3\end{array}\right]^{T}, \mathbf{g}_{2}=\frac{1}{2}\left[\begin{array}{ll}1 & 1\end{array}\right]^{T}$. One can easily confirm that in this example, $\beta_{1}=\frac{\pi}{3}$ and $\beta_{2}=\frac{\pi}{4}$. Fig. 3 shows the track of NE points when $P_{2}$ changes from 0.01 to 58.75. For each $P_{2}$, we obtain the optimal NE strategies from (39), (40), and consequently calculate the secrecy rates at the NE point as given by (41). The direction of the arrows shows how the NE points change as $P_{2}$ increases; when $P_{2}=0.01$ the NE is at point $A$ and when $P_{2}=58.75$ the NE is at point $C$. The game provides a maximum secrecy rate for Tx2 when $P_{2}=3.94$, corresponding to point $B$ on the curve. However, we see that increasing $P_{2}$ beyond 3.94 results in the game providing a lower secrecy rate for $\mathrm{T} \times 2$. In this case, a greater secrecy advantage

\footnotetext{
${ }^{2}$ Note that the obtained analytical results are valid for arbitrary channels with complex gains and an arbitrary number of antennas at the transmitters. The simple channels chosen for this example are just for ease of illustration.
} 


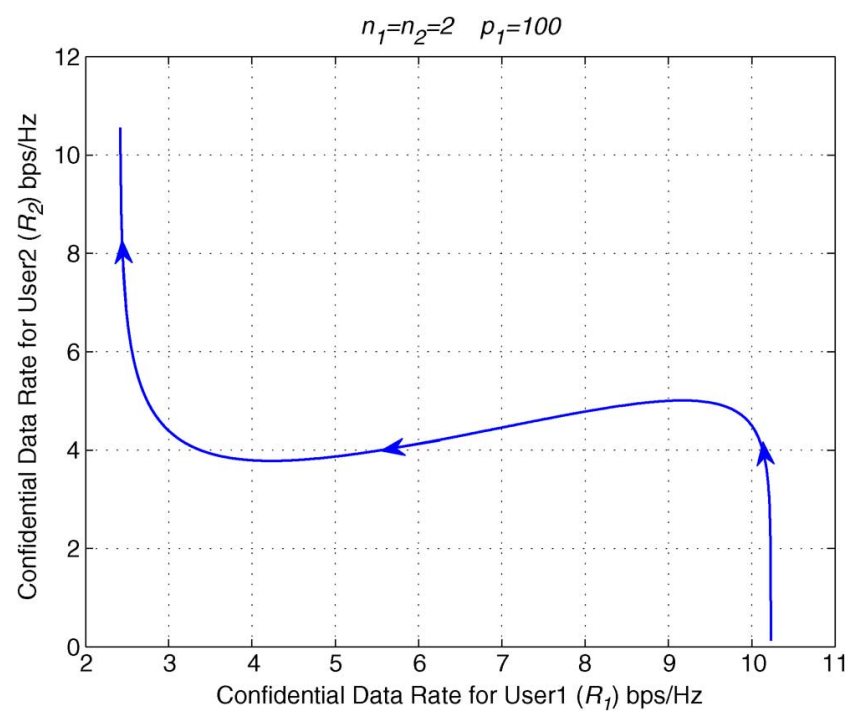

Fig. 4. Track of NE points in response to the changes of $P_{2}$ from 0.01 to 5000 , when $\beta_{2}=\frac{\pi}{2} \cdot \mathbf{h}_{1}=\left[\begin{array}{ll}4 & 0\end{array}\right]^{T}, \mathbf{g}_{1}=[\sqrt{33}]^{T}, \mathbf{h}_{2}=\left[\begin{array}{ll}0 & 3\end{array}\right]^{T}, \mathbf{g}_{2}=[\sqrt{20}]^{T}$.

for Tx2, measured in terms of $R_{2}-R_{1}$, is obtained by using additional power at $\mathrm{T} \times 2$ to reduce the secrecy rate achieved by Tx1. One can verify that $R_{2}-R_{1}$ is monotonically increasing with $P_{2}$, even if $R_{2}$ is not. For $P_{2}>58.75$, the game results in $R_{2}=0$, and one would have to consider an alternative strategy space for Tx 2 that included jamming Tx1 in order to further reduce $R_{1}$.

In the next example, Fig. 4, we retain the system parameters of the first example, except $\mathrm{g}_{2}=[\sqrt{2} 0]^{T}$, which results in $\mathbf{h}_{2} \perp \mathbf{g}_{2}$, i.e., $\beta_{2}=\frac{\pi}{2}$. Here $P_{2}$ changes from 0.01 to 5000 . This example is of special interest because when $\beta_{2}=\frac{\pi}{2}$, we have $\theta_{2}^{*}=\frac{\pi}{2}$, as given by (40) and mentioned in Section III-C-3. In other words, for any $P_{2}$, the best that Tx 2 can do in maximizing $R_{2}-R_{1}$ is to simply maximize its secrecy rate $R_{2}$ by using a beamformer orthogonal to $\mathbf{g}_{2}$, i.e., $\mathbf{w}_{2}^{*}=\frac{\mathbf{h}_{2}}{\left\|\mathbf{h}_{2}\right\|}$. When $P_{2}$ is very large, the NE appears to converge to a point where the secrecy rate $R_{1}$ is fixed and independent of $P_{2}$. This is due to the fact that, when $\theta_{2}^{*}=\frac{\pi}{2}$, function $\mathcal{F}\left(=R_{1}-R_{2}\right)$ is given by

$$
\begin{aligned}
\mathcal{F}=\log \left(1+P_{1} \mathbf{h}_{1}^{H}\right. & \left.\mathbf{h}_{1} \cos ^{2}\left(\theta_{1}-\beta_{1}\right)\right) \\
& -\log \left(1+P_{2} \mathbf{h}_{2}^{H} \mathbf{h}_{2}+P_{1} \mathbf{g}_{1}^{H} \mathbf{g}_{1} \cos ^{2}\left(\theta_{1}\right)\right)
\end{aligned}
$$

Clearly, as $P_{2} \rightarrow \infty$, the choice of $\theta_{1}$ cannot considerably affect the second $\log$ term. Thus, when $P_{2} \rightarrow \infty$, the best that Tx 1 can do is maximize the first $\log$ term by choosing $\theta_{1}^{*}=\beta_{1}$, i.e., $\mathbf{w}_{1}^{*}=\frac{\mathbf{h}_{1}}{\left\|\mathbf{h}_{1}\right\|}$.

The above point is an important observation which completes the discussion in Section III-C-3. When $\beta_{2}=\frac{\pi}{2}\left(\mathbf{h}_{2} \perp \mathbf{g}_{2}\right)$ and $P_{2} \rightarrow \infty$, the optimal NE strategy at both transmitters is given by $\theta_{i}^{*}=\beta_{i}$ or $\mathbf{w}_{i}^{*}=\frac{\mathbf{h}_{i}}{\left\|\mathbf{h}_{i}\right\|}, i=1,2$. Clearly, the same statement holds for the case that $\beta_{1}=\frac{\pi}{2}$ and $P_{1} \rightarrow \infty$. Consequently, the $\mathrm{NE}$ point $\left(R_{1}, R_{2}\right)$ is given by

$$
\begin{aligned}
& R_{1}=\log \left(1+P_{1} \mathbf{h}_{1}^{H} \mathbf{h}_{1}\right)-\log \left(1+P_{1} \mathbf{g}_{1}^{H} \mathbf{g}_{1} \cos ^{2}\left(\beta_{1}\right)\right) \\
& R_{2}=\log \left(1+P_{2} \mathbf{h}_{2}^{H} \mathbf{h}_{2}\right) .
\end{aligned}
$$

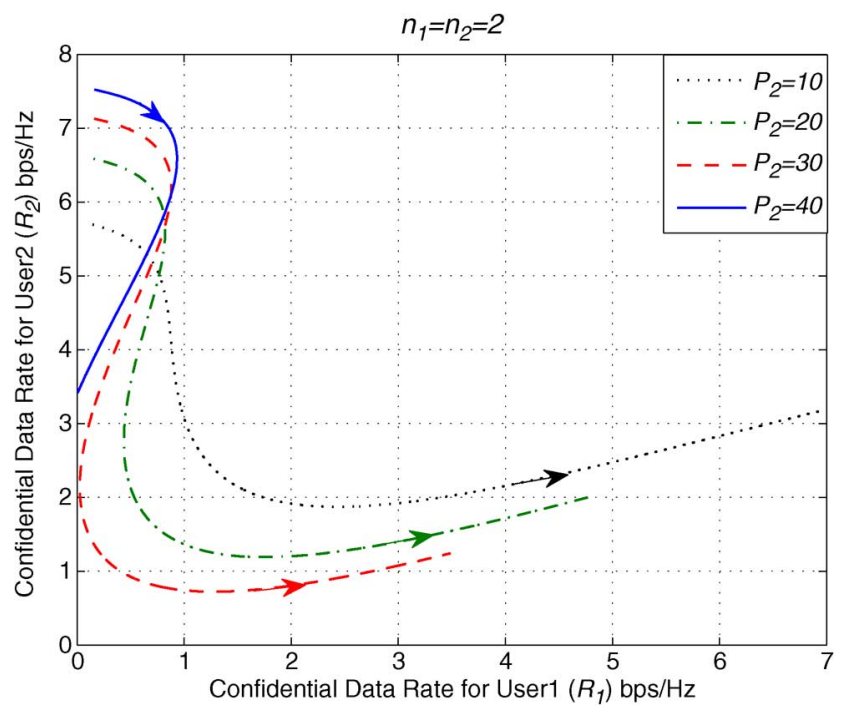

Fig. 5. Track of NE points in response to the changes of $P_{1}$ from 0.01 to 100 , for different $P_{2}$ values, $\mathbf{h}_{1}=\left[\begin{array}{ll}4 & 0\end{array}\right]^{T}, \mathbf{g}_{1}=[\sqrt{33}]^{T}, \mathbf{h}_{2}=\left[\begin{array}{ll}0 & 3\end{array}\right]^{T}, \mathbf{g}_{2}=$ $\frac{1}{2}\left[\begin{array}{ll}1 & 1\end{array}\right]^{T}$.

However it should be noted that, while for $\beta_{2}=\frac{\pi}{2}$ the optimal NE strategy at Tx2 always results in a secrecy rate $R_{2}>$ 0 when $P_{2} \rightarrow \infty$, if $\left\|\mathbf{h}_{1}\right\|<\left\|\mathbf{g}_{1}\right\| \cos \left(\beta_{1}\right)$ or equivalently $\mathbf{h}_{1}^{H} \mathbf{h}_{1}<\left|\mathbf{h}_{1}^{H} \mathbf{g}_{1}\right| \mid$, this game would result in $R_{1}=0$, and since the problem would no longer be an IFC, the NE strategies $\mathbf{w}_{i}^{*}=\frac{\mathbf{h}_{i}}{\left\|\mathbf{h}_{i}\right\|}$ would no longer be valid.

In the next example, Fig. 5, we consider the joint effect of $P_{1}$ and $P_{2}$ on the Nash equilibrium point. The channel vectors are the same as those in the first example. In Fig. 5, each curve corresponds to a given $P_{2}$ and shows the track of NE points when $P_{1}$ changes from 0.01 to 100 . The direction of the arrows shows how the NE points change as $P_{1}$ increases. The figure shows that for $P_{2}=40$, Tx1 has to shut down to maximize $R_{1}-R_{2}$ when $P_{1}>1.64$. The figure shows that, as expected, for any $P_{2}, R_{1}-R_{2}$ increases with $P_{1}$. Similarly, for a given $P_{1}, R_{2}-R_{1}$ increases with $P_{2}$.

In Fig. 6, we consider the joint effect of $\beta_{2}$ and $P_{1}$ on the NE point. We assume $P_{2}=10$ and $\mathbf{g}_{2}=\frac{\sqrt{2}}{2}\left[\cos \left(\beta_{2}\right) \sin \left(\beta_{2}\right)\right]^{T}$, where $\beta_{2}$ changes from 0 to $\frac{\pi}{2}$. The other channel vectors are unchanged from the first example. Each curve corresponds to a given value of $P_{1}$, and the direction of the arrows shows how the NE points change when $\beta_{2}$ is increased from 0 to $\frac{\pi}{2}$. We see that for this example, changing $\beta_{2}$ causes the NE points to move on a line whose slope appears to be independent of $P_{1}$. The slope of the line is greater than one, so while both $R_{1}$ and $R_{2}$ increase, $R_{2}-R_{1}$ increases as well.

In Fig. 7, we compute the average NE points using (41) for Rayleigh fading channels, where the entries of the channel vectors are independent and distributed as $\mathcal{C N}(0,1)$. In this example we assume $P_{1}=10$ and $P_{2}=100$. The figure shows the location of the average NE points over 10000 realization as $n_{1}$ changes from 2 to 8 and either $n_{2}=2$ or $n_{2}=n_{1}$. The direction of the arrows shows how the NE points change as $n_{1}$ increases. As $n_{1}$ increases when $n_{2}=2$ is fixed, the secrecy rate at the NE point improves for Tx 1 and decreases for Tx 2 . When the number of antennas at both transmitters increases, the achieved 


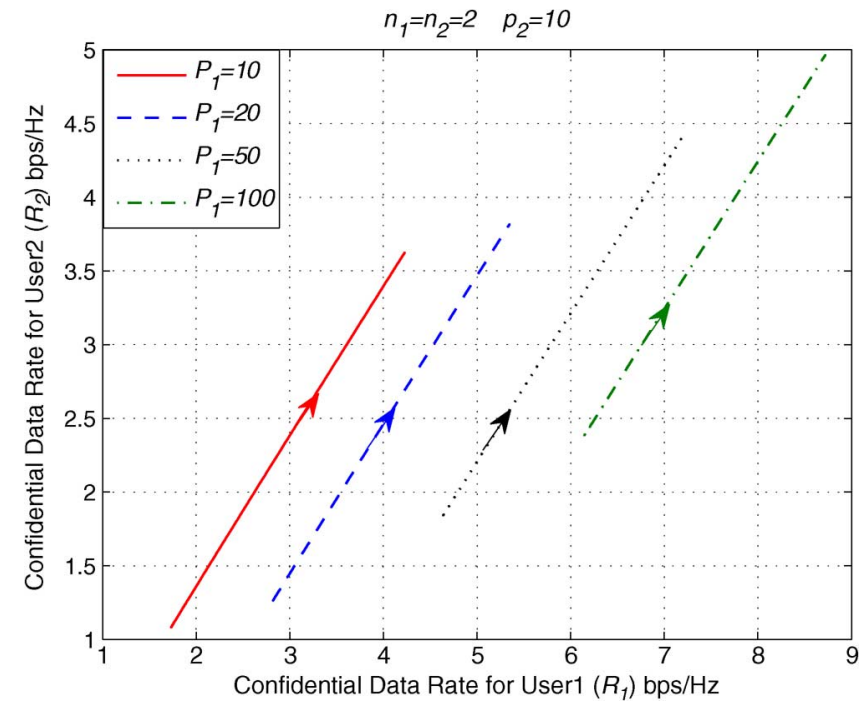

Fig. 6. Track of NE points in response to the changes of $\beta_{2}$ from 0 to $\frac{\pi}{2}$ for different $P_{1}$ values, $\mathbf{h}_{1}=\left[\begin{array}{ll}4 & 0\end{array}\right]^{T}, \mathbf{g}_{1}=[\sqrt{33}]^{T}, \mathbf{h}_{2}=\left[\begin{array}{ll}0 & 3\end{array}\right]^{T}, \mathbf{g}_{2}=$ $\frac{\sqrt{2}}{2}\left[\cos \left(\beta_{2}\right) \sin \left(\beta_{2}\right)\right]^{T}$.

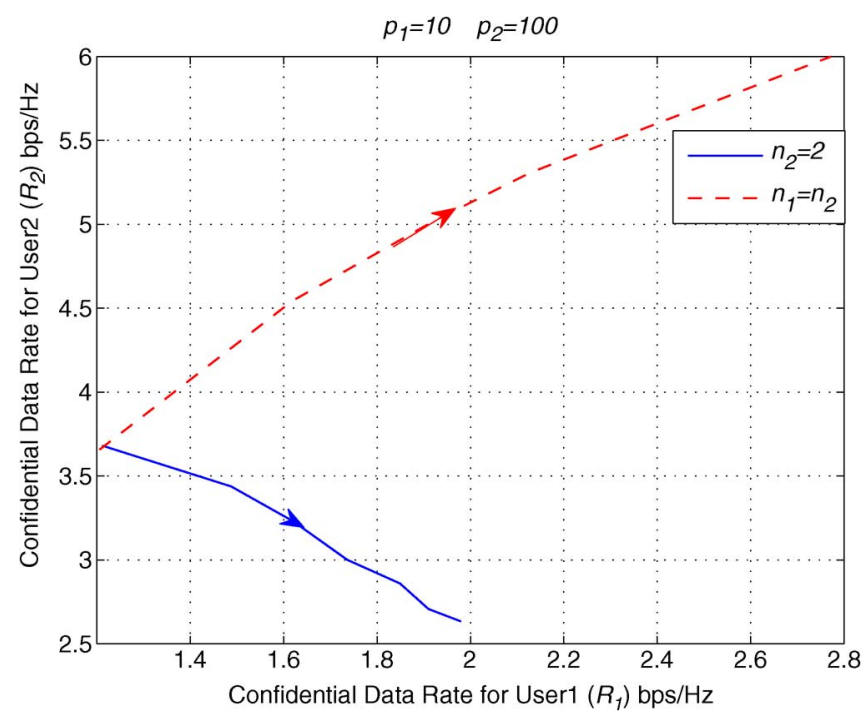

Fig. 7. Track of average NE points, for Rayleigh fading channels, in response to the changes of $n_{1}$ from 2 to 8 for different $n_{2}$ scenarios. $n_{2}=2$ and $n_{2}=n_{1}$.

secrecy rate at the NE point for both transmitters increases as well.

Fig. 8 shows the average number of iterations for the algorithm in Section III-D to converge to the NE point for the previous example. Here we set the convergence tolerance as $\epsilon=10^{-4}$. As the figure shows, even when the number of antennas at the transmitters is large, the iterative algorithm almost always converges in less than 3 iterations. Interestingly, the figure shows that when $n_{1}=n_{2}$, the average number of iterations for convergence is less than when $n_{2}=2$.

Finally Fig. 9 compares NE operating points for different applications, where the channel parameters are: $\mathbf{h}_{1}=[0.19-$ $0.16 i 0.02+0.60 i]^{T}, \mathbf{g}_{1}=[0.62+0.51 i-0.29-0.43 i]^{T}$, $\mathbf{h}_{2}=[0.19+0.70 i 0.54+0.69 i]^{T}$, and $\mathbf{g}_{2}=[-0.56-$ $0.03 i 0.08+0.73 i]^{T}$. The figure shows the achievable rate (red) and secrecy rate (blue) regions [23], as well as a sample of

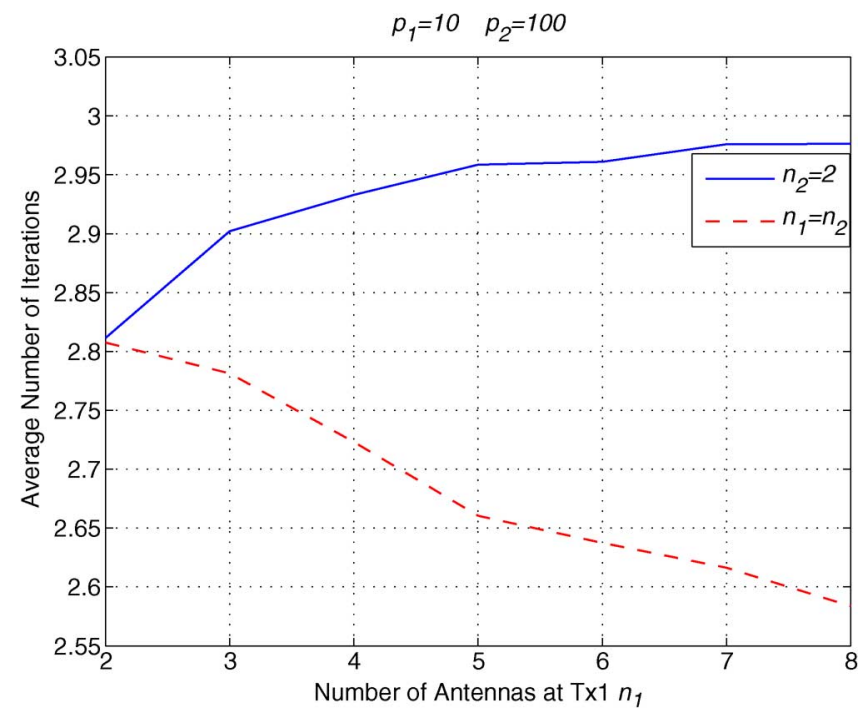

Fig. 8. Average number of iterations for the iterative algorithm to converge to the NE points, for Rayleigh fading channels, in response to the changes of $n_{1}$ from 2 to 8 for different $n_{2}$ scenarios. $n_{2}=2$ and $n_{2}=n_{1}$.

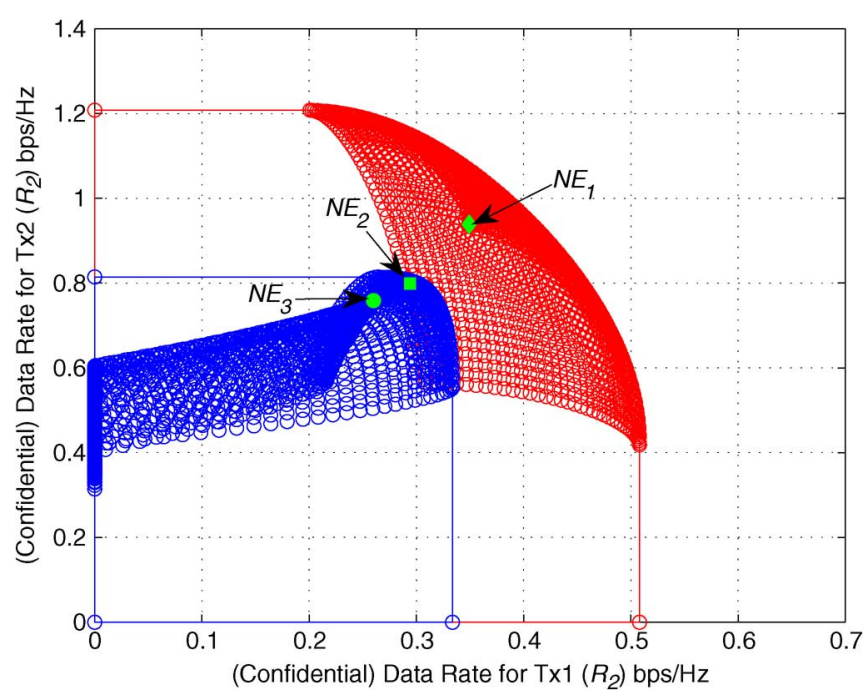

Fig. 9. Comparison different NE points for different application, as well as the achievable rate and secrecy rate regions.

achievable rate points within each region denoted by red and blue circles. The axes show either the achieved rate or secrecy rate for each user, depending on whether or not secrecy constraints are considered. The figure also shows the location of three key NE operating points: the NE point without secrecy constraints $\left(N E_{1}\right)$, where each transmitter wants to maximize its own rate $^{3}$, the NE point where each transmitter wants to maximize its own secrecy rate $\left(N E_{2}\right)$, and the NE point proposed in this paper where each transmitter aims to maximize the difference between its own secrecy rate and the secrecy rate of the other link $\left(N E_{3}\right)$. To obtain $N E_{2}$, one can use the iterative algorithm proposed in [16]. Note that the fact that $N E_{2}$ dominates $N E_{3}$ does not imply that $N E_{3}$ is an "inferior" operating point,

\footnotetext{
${ }^{3}$ Clearly, for this scenario the NE strategy at each transmitter is maximum ratio transmission (MRT) with $\mathbf{w}_{i}=\frac{\mathbf{h}_{i}}{\left\|\mathbf{h}_{i}\right\|}[23]$.
} 
since the two equilibria are based on different definitions of the utility function.

\section{CONCLUSION}

In this paper, we have characterized the Nash Equilibrium solution for the MISO Gaussian interference channel where each link aims to maximize the difference between its secrecy rate and the secrecy rate of the other link. We showed that when the transmitters employ Gaussian signaling, the game has a unique Nash equilibrium based on the use of beamforming at the transmitters. We obtained closed-form solutions for the beamformers employed by the transmitters at the NE point, and illustrated the resulting secrecy rate behavior via several numerical examples. While the NE can be found in one shot when full CSI is available to the transmitters, we also showed that an iterative algorithm can converge to the NE when only limited CSI is available.

\section{APPENDIX A}

\section{PROOF OF THEOREM 1}

We are interested in critical points of $\mathcal{F}$ in the range of $\left(\theta_{1}, \theta_{2}\right) \in\left\{\left[0, \frac{\pi}{2}\right] \times\left[0, \frac{\pi}{2}\right]\right\}$. By definition, to obtain the critical point $\left(\bar{\theta}_{1}, \hat{\theta}_{2}\right)$, we must solve the equations $\frac{\partial \mathcal{F}}{\partial \theta_{1}}=0$ and $\frac{\partial \mathcal{F}}{\partial \theta_{2}}=0$ simultaneously. The following equations are considered for $\bar{\theta}_{i}$ but for notational simplicity we drop the "-_. from $\bar{\theta}_{i}$.

By applying $\frac{\partial \mathcal{F}}{\partial \theta_{i}}=0$ to (26), we have

$$
\begin{aligned}
& \frac{h_{1} \sin 2\left(\theta_{1}-\beta_{1}\right)}{1+h_{1} \cos ^{2}\left(\theta_{1}-\beta_{1}\right)+g_{2} \cos ^{2}\left(\theta_{2}\right)} \\
& =\frac{g_{1} \sin \left(2 \theta_{1}\right)}{1+h_{2} \cos ^{2}\left(\theta_{2}-\beta_{2}\right)+g_{1} \cos ^{2}\left(\theta_{1}\right)} \\
& \frac{g_{2} \sin \left(2 \theta_{2}\right)}{1+h_{1} \cos ^{2}\left(\theta_{1}-\beta_{1}\right)+g_{2} \cos ^{2}\left(\theta_{2}\right)} \\
& =\frac{h_{2} \sin 2\left(\theta_{2}-\beta_{2}\right)}{1+h_{2} \cos ^{2}\left(\theta_{2}-\beta_{2}\right)+g_{1} \cos ^{2}\left(\theta_{1}\right)} .
\end{aligned}
$$

Define $m$ as

$$
m=\frac{1+h_{1} \cos ^{2}\left(\theta_{1}-\beta_{1}\right)+g_{2} \cos ^{2}\left(\theta_{2}\right)}{1+h_{2} \cos ^{2}\left(\theta_{2}-\beta_{2}\right)+g_{1} \cos ^{2}\left(\theta_{1}\right)} .
$$

Using (43)-(45) we have:

$$
\frac{h_{1} \sin 2\left(\theta_{1}-\beta_{1}\right)}{g_{1} \sin \left(2 \theta_{1}\right)}=\frac{g_{2} \sin \left(2 \theta_{2}\right)}{h_{2} \sin 2\left(\theta_{2}-\beta_{2}\right)}=m,
$$

and (46) leads to

$$
\begin{aligned}
\tan \left(2 \theta_{1}\right) & =\frac{\sin \left(2 \beta_{1}\right)}{\cos \left(2 \beta_{1}\right)-\frac{g_{1}}{h_{1}} m} \\
\tan \left(2 \theta_{2}\right) & =\frac{\sin \left(2 \beta_{2}\right)}{\cos \left(2 \beta_{2}\right)-\frac{g_{2}}{h_{2}} \frac{1}{m}} .
\end{aligned}
$$

Using $\tan (x)=\frac{1-\cos (2 x)}{\sin (2 x)}$, we can write

$$
\begin{aligned}
& \tan \left(\theta_{1}\right) \\
& =\left(\frac{\sqrt{1-2 \cos \left(2 \beta_{1}\right) \frac{g_{1}}{h_{1}} m+\frac{g_{1}^{2}}{h_{1}^{2}} m^{2}}-\cos \left(2 \beta_{1}\right)+\frac{g_{1}}{h_{1}} m}{\sin \left(2 \beta_{1}\right)}\right) \\
& \tan \left(\theta_{2}\right) \\
& =\left(\frac{\sqrt{1-2 \cos \left(2 \beta_{2}\right) \frac{g_{2}}{h_{2}} \frac{1}{m}+\frac{g_{2}^{2}}{h_{2}^{2}} \frac{1}{m^{2}}}-\cos \left(2 \beta_{2}\right)+\frac{g_{2}}{h_{2}} \frac{1}{m}}{\sin \left(2 \beta_{2}\right)}\right),
\end{aligned}
$$

which clearly prove (29) and (30), respectively.

Note that the only unknown parameter in characterizing $\left(\bar{\theta}_{1}, \bar{\theta}_{2}\right)$ is $m$. Using (47) and (48) in (45), and after some basic algebraic calculations, we have

$$
\begin{aligned}
& 1+\frac{g_{2}}{2}+\frac{h_{1}}{2}\left(1+\sqrt{1-2 \cos \left(2 \beta_{1}\right) \frac{g_{1}}{h_{1}} m+\frac{g_{1}^{2}}{h_{1}^{2}} m^{2}}\right)=m \\
& +m \frac{g_{1}}{2}+m \frac{h_{2}}{2}\left(1+\sqrt{1-2 \cos \left(2 \beta_{2}\right) \frac{g_{2}}{h_{2}} \frac{1}{m}+\frac{g_{2}^{2}}{h_{2}^{2}} \frac{1}{m^{2}}}\right) .
\end{aligned}
$$

We now show that (51) has only one real positive solution. Define $\mathcal{G}(m)$ as

$$
\begin{array}{r}
\mathcal{G}(m)=m+m \frac{g_{1}}{2}+m \frac{h_{2}}{2}+\frac{h_{2}}{2} \sqrt{m^{2}-2 \cos \left(2 \beta_{2}\right) \frac{g_{2}}{h_{2}} m+\frac{g_{2}^{2}}{h_{2}^{2}}} \\
-1-\frac{g_{2}}{2}-\frac{h_{1}}{2}\left(1+\sqrt{1-2 \cos \left(2 \beta_{1}\right) \frac{g_{1}}{h_{1}} m+\frac{g_{1}^{2}}{h_{1}^{2}} m^{2}}\right),
\end{array}
$$

and note that $\mathcal{G}(0)=-\left(1+h_{1}\right)<0$. Moreover, $\mathcal{G}(m)$ is monotonically increasing:

$$
\begin{aligned}
\frac{\partial \mathcal{G}}{\partial m}= & 1+\frac{g_{1}}{2}+\frac{h_{2}}{2}+\frac{h_{2}}{2} \frac{m-\cos \left(2 \beta_{2}\right) \frac{g_{2}}{h_{2}}}{\sqrt{m^{2}-2 \cos \left(2 \beta_{2}\right) \frac{g_{2}}{h_{2}} m+\frac{g_{2}^{2}}{h_{2}^{2}}}} \\
& +\frac{g_{1}}{2} \frac{\cos \left(2 \beta_{1}\right)-\frac{g_{1}}{h_{1}} m}{\sqrt{1-2 \cos \left(2 \beta_{1}\right) \frac{g_{1}}{h_{1}} m+\frac{g_{1}^{2}}{h_{1}^{2}} m^{2}}} \\
= & 1+\frac{g_{1}}{2}+\frac{h_{2}}{2}+\frac{h_{2}}{2} \cos 2\left(\theta_{2}-\beta_{2}\right)+\frac{g_{1}}{2} \cos \left(2 \theta_{1}\right) \\
= & 1+\frac{g_{1}}{2}\left(1+\cos \left(2 \theta_{1}\right)\right)+\frac{h_{2}}{2}\left(1+\cos 2\left(\theta_{2}-\beta_{2}\right)\right) \\
\geq & 1>0 .
\end{aligned}
$$

This establishes the fact that there exists one and only one $m>$ 0 for which $\mathcal{G}(m)=0$.

From (51), via straightforward computation including squaring both sides of (51), rearranging and squaring again, we get the following polynomial equation for $m$ :

$$
a m^{4}+b m^{3}+c m^{2}+d m+e=0
$$


where

$$
\begin{aligned}
a= & A^{2}-\frac{1}{4} g_{1}^{2} h_{2}^{2} \\
b= & -2 A B+\frac{1}{2} g_{1}^{2} h_{2} g_{2} \cos \left(2 \beta_{2}\right)+\frac{1}{2} h_{2}^{2} h_{1} g_{1} \cos \left(2 \beta_{1}\right) \\
c= & B^{2}+2 A C-\frac{1}{4} h_{1}^{2} h_{2}^{2}-\frac{1}{4} g_{1}^{2} g_{2}^{2} \\
& -g_{1} g_{2} h_{1} h_{2} \cos \left(2 \beta_{1}\right) \cos \left(2 \beta_{2}\right) \\
d= & -2 C B+\frac{1}{2} g_{2}^{2} h_{1} g_{1} \cos \left(2 \beta_{1}\right)+\frac{1}{2} h_{1}^{2} h_{2} g_{2} \cos \left(2 \beta_{2}\right) \\
e= & C^{2}-\frac{1}{4} g_{2}^{2} h_{1}^{2}
\end{aligned}
$$

and

$$
\begin{aligned}
A= & 1+g_{1}+h_{2}+\frac{1}{2} g_{1} h_{2} \\
B= & 2+g_{1}+g_{2}+h_{1}+h_{2}+\frac{1}{2} h_{1} h_{2}+\frac{1}{2} g_{1} g_{2} \\
& +g_{2} h_{2} \sin ^{2}\left(\beta_{2}\right)+g_{1} h_{1} \sin ^{2}\left(\beta_{1}\right) \\
C= & 1+g_{2}+h_{1}+\frac{1}{2} g_{2} h_{1} .
\end{aligned}
$$

Note that the polynomial coefficients in (52)-(56) alternately change sign; in particular, we have $a>0, b<0, c>0$, $d<0$ and $e>0$. Thus, using Sturm's theorem, we know that the polynomial has four real positive solutions. The root of interest is the one that satisfies (51). This completes the proof of Theorem 1.

\section{APPENDIX B}

\section{PROOF OF THEOREM 2}

We want to prove that

$$
\bar{\theta}_{1}=\arg \max _{\theta_{1}} \mathcal{F}\left(\theta_{1}, \bar{\theta}_{2}\right) .
$$

One can easily confirm that

$$
\begin{aligned}
\arg \max _{\theta_{1}} \mathcal{F}\left(\theta_{1}, \bar{\theta}_{2}\right) & =\arg \max _{\theta_{1}} \log \left(1+\frac{h_{1} \cos ^{2}\left(\theta_{1}-\beta_{1}\right)}{1+g_{2} \cos ^{2}\left(\bar{\theta}_{2}\right)}\right) \\
& -\log \left(1+\frac{g_{1} \cos ^{2}\left(\theta_{1}\right)}{1+h_{2} \cos ^{2}\left(\bar{\theta}_{2}-\beta_{2}\right)}\right) .
\end{aligned}
$$

Now reverting the problem to its vector representation, by applying (22)-(25) in (58), we have

$$
\begin{aligned}
\max _{\theta_{1}} & \log \left(1+\frac{h_{1} \cos ^{2}\left(\theta_{1}-\beta_{1}\right)}{1+g_{2} \cos ^{2}\left(\bar{\theta}_{2}\right)}\right) \\
& -\log \left(1+\frac{g_{1} \cos ^{2}\left(\theta_{1}\right)}{1+h_{2} \cos ^{2}\left(\bar{\theta}_{2}-\beta_{2}\right)}\right) \\
= & \max _{\mathbf{w}_{1}} \log \left(1+\frac{\mathbf{h}_{1}^{H} \mathbf{w}_{1} \mathbf{w}_{1}^{H} \mathbf{h}_{1}}{1+g_{2} \cos ^{2}\left(\bar{\theta}_{2}\right)}\right) \\
& -\log \left(1+\frac{\mathbf{g}_{1}^{H} \mathbf{w}_{1} \mathbf{w}_{1}^{H} \mathbf{g}_{1}}{1+h_{2} \cos ^{2}\left(\bar{\theta}_{2}-\beta_{2}\right)}\right) .
\end{aligned}
$$

Eq. (59) is a Rayleigh quotient problem, and the solution for $\mathbf{w}_{1}$ is the normalized principal generalized eigenvector corresponding to the largest generalized eigenvalue of the following pencil

$$
\left(\mathbf{I}+\frac{1}{1+g_{2} \cos ^{2}\left(\bar{\theta}_{2}\right)} \mathbf{h}_{1} \mathbf{h}_{1}^{H}, \mathbf{I}+\frac{1}{1+h_{2} \cos ^{2}\left(\bar{\theta}_{2}-\beta_{2}\right)} \mathbf{g}_{1} \mathbf{g}_{1}^{H}\right)
$$

Lemma 3: For any two vectors $\mathbf{h}$ and $\mathbf{g}$ of the same dimension, the principal generalized eigenvector of $\left(\mathbf{I}+\mathbf{h h}^{H}, \mathbf{I}+\right.$ $\mathrm{gg}^{H}$ ) is given by

$$
\mathbf{w}_{t}=\frac{\left(-u+\sqrt{u^{2}+4 v r}\right) \mathbf{P}_{g}^{\perp} \mathbf{h}+2 r \mathbf{P}_{g} \mathbf{h}}{\left\|\left(-u+\sqrt{u^{2}+4 v r}\right) \mathbf{P}_{g}^{\perp} \mathbf{h}+2 r \mathbf{P}_{g} \mathbf{h}\right\|}
$$

where $u=\left(1+\mathbf{h}^{H} \mathbf{P}_{g} \mathbf{h}\right)-\left(\mathbf{g}^{H} \mathbf{g}+1\right)\left(1+\mathbf{h}^{H} \mathbf{P}_{g}^{\perp} \mathbf{h}\right), r=$ $\left(\mathbf{h}^{H} \mathbf{P}_{g}^{\perp} \mathbf{h}\right)$, and $v=\left(\mathbf{g}^{H} \mathbf{g}+1\right)\left(\mathbf{h}^{H} \mathbf{P}_{g} \mathbf{h}\right)$.

Proof: From the definition of the generalized eigenvalue decomposition we have [25]:

$$
\left(\mathbf{I}+\mathbf{h h}^{H}\right) \mathbf{w}_{t}=\lambda_{t}\left(\mathbf{I}+\mathbf{g g}^{H}\right) \mathbf{w}_{t} .
$$

It is easy to verify that among the $n_{t}$ generalized eigenvalues of the pencil $\left(\mathbf{I}+\mathbf{h h}^{H}, \mathbf{I}+\mathbf{g g}^{H}\right),\left(n_{t}-2\right)$ of them are equal to 1 and are obtained using any vector that is orthogonal to the space spanned by [hg]. The two generalized eigenvectors corresponding to the maximum $\left(\lambda_{t}\right)$ and the minimum generalized eigenvalues lie completely in $\operatorname{span}([\mathbf{h g}])$. Thus, we can write $\mathbf{w}_{t}$ as

$$
\mathbf{w}_{t}=\nu_{1} \mathbf{P}_{g}^{\perp} \mathbf{h}+\nu_{2} \mathbf{P}_{g} \mathbf{h}
$$

for some complex numbers $\nu_{1}$ and $\nu_{2}$ that must be solved for, where $\mathbf{P}_{g}=\left(\mathbf{g}^{H} \mathbf{g}\right)^{-1} \mathbf{g g}^{H}$ denotes the orthogonal projection onto $\operatorname{span}(\mathbf{g})$ and $\mathbf{P}_{g}^{\perp}=\mathbf{I}-\mathbf{P}_{g}$ denotes the projection onto its orthogonal complement. From (61), we have

$$
\left(\mathbf{I}+\mathbf{g g}^{H}\right)^{-1}\left(\mathbf{I}+\mathbf{h h}^{H}\right) \mathbf{w}_{t}=\lambda_{t} \mathbf{w}_{t} .
$$

Note that $\left(\mathbf{I}+\mathbf{g g}^{H}\right)^{-1}=\left(\mathbf{P}_{g}^{\perp}+\left(\mathbf{g}^{H} \mathbf{g}+1\right)^{-1} \mathbf{P}_{g}\right)$. Now it is easy to verify that

$$
\mathbf{w}_{t}=\frac{\left(-u+\sqrt{u^{2}+4 v r}\right) \mathbf{P}_{g}^{\perp} \mathbf{h}+2 r \mathbf{P}_{g} \mathbf{h}}{\left\|\left(-u+\sqrt{u^{2}+4 v r}\right) \mathbf{P}_{g}^{\perp} \mathbf{h}+2 r \mathbf{P}_{g} \mathbf{h}\right\|}
$$

and

$$
\lambda_{t}=\frac{\left(1+\mathbf{h}^{H} \mathbf{P}_{g} \mathbf{h}\right)+\left(1+\mathbf{h}^{H} \mathbf{P}_{g}^{\perp} \mathbf{h}\right)\left(\mathbf{g}^{H} \mathbf{g}+1\right)+\sqrt{u^{2}+4 r v}}{\left(\mathbf{g}^{H} \mathbf{g}+1\right)}
$$

where $u=\left(1+\mathbf{h}^{H} \mathbf{P}_{g} \mathbf{h}\right)-\left(\mathbf{g}^{H} \mathbf{g}+1\right)\left(1+\mathbf{h}^{H} \mathbf{P}_{g}^{\perp} \mathbf{h}\right), r=$ $\left(\mathbf{h}^{H} \mathbf{P}{ }_{g}^{\perp} \mathbf{h}\right)$, and $v=\left(\mathbf{g}^{H} \mathbf{g}+1\right)\left(\mathbf{h}^{H} \mathbf{P}_{g} \mathbf{h}\right)$. This completes the proof of Lemma 3. 


$$
\begin{aligned}
\widehat{\theta}_{1} & =\tan ^{-1}\left(\frac{-u+\sqrt{u^{2}+4 v r}}{2 r} \tan \left(\beta_{1}\right)\right) \\
& =\tan ^{-1}\left(\frac{-\cos \left(2 \beta_{1}\right)+p+\sqrt{\left(\cos \left(2 \beta_{1}\right)-p\right)^{2}+(1+q) \sin ^{2}\left(2 \beta_{1}\right)}}{\sin \left(2 \beta_{1}\right)}\right),
\end{aligned}
$$

Now by applying Lemma 3 on the pencil (60), one can confirm that the optimal $\mathbf{w}_{1}$ that maximizes (59) is given by

$$
\begin{aligned}
\widehat{\mathbf{w}}_{1} & =\frac{\left(-u+\sqrt{u^{2}+4 v r}\right) \mathbf{P}_{g_{1}}^{\perp} \mathbf{h}_{1}+2 r \mathbf{P}_{g_{1}} \mathbf{h}_{1}}{\left\|\left(-u+\sqrt{u^{2}+4 v r}\right) \mathbf{P}_{g_{1}}^{\perp} \mathbf{h}_{1}+2 r \mathbf{P}_{g_{1}} \mathbf{h}_{1}\right\|} \\
& =\cos \left(\widehat{\theta}_{1}\right) \frac{\mathbf{P}_{g_{1}} \mathbf{h}_{1}}{\left\|\mathbf{P}_{g_{1}} \mathbf{h}_{1}\right\|}+\sin \left(\widehat{\theta}_{1}\right) \frac{\mathbf{P}_{g_{1}}^{\perp} \mathbf{h}_{1}}{\left\|\mathbf{P}_{g_{1}}^{\perp} \mathbf{h}_{1}\right\|}
\end{aligned}
$$

where, for example, $v$ becomes

$$
v=\left(\frac{g_{1}}{1+h_{2} \cos ^{2}\left(\bar{\theta}_{2}-\beta_{2}\right)}+1\right) \frac{\mathbf{h}_{1}^{H} \mathbf{P}_{g_{1}} \mathbf{h}_{1}}{1+g_{2} \cos ^{2}\left(\bar{\theta}_{2}\right)} .
$$

Going back to problem (58), it is clear that $\widehat{\theta}_{1}$, corresponding to $\widehat{\mathbf{w}}_{1}$, is the optimal $\theta_{1}$ that maximizes $\mathcal{F}\left(\theta_{1}, \bar{\theta}_{2}\right)$ and is given by (63) at the top of the page, where $p=\frac{g_{1}}{h_{1}} \frac{1+g_{2} \cos ^{2}\left(\bar{\theta}_{2}\right)+h_{1}}{1+h_{2} \cos ^{2}\left(\bar{\theta}_{2}-\beta_{2}\right)}$ and $q=\frac{g_{1}}{1+h_{2} \cos ^{2}\left(\bar{\theta}_{2}-\beta_{2}\right)} \cdot \operatorname{In}(63)$, we used the fact that $\mathbf{h}_{1}^{H} \mathbf{P}_{g_{1}}^{\perp} \mathbf{h}_{1}=$ $h_{1} \sin ^{2}\left(\beta_{1}\right)$ and $\mathbf{h}_{1}^{H} \mathbf{P}_{g_{1}} \mathbf{h}_{1}-\mathbf{h}_{1}^{H} \mathbf{P}_{g_{1}}^{\perp} \mathbf{h}_{1}=h_{1} \cos \left(2 \beta_{1}\right)$, where $\beta_{i}, h_{i}$ and $g_{i}$ are defined in (21)-(25).

Note that, besides the system parameters $h_{i}, g_{i}$ and $\beta_{i}, \widehat{\theta}_{1}$ in (63) is a function of $\bar{\theta}_{2}$ which itself is a function of the realvalued parameter $m$ obtained in Theorem 1. Recall that the main purpose of this appendix is to prove (57); i.e., we must show that for the $m$ given in Theorem $1, \bar{\theta}_{1}=\widehat{\theta}_{1}$, where $\bar{\theta}_{1}$ is given by (29). Comparing (29) and (63), it is clear that to prove $\bar{\theta}_{1}=\widehat{\theta}_{1}$, one must show the following equality holds for the value of $m$ obtained in Theorem 1:

$$
\begin{aligned}
& p+\sqrt{\left(\cos \left(2 \beta_{1}\right)-p\right)^{2}+(1+q) \sin ^{2}\left(2 \beta_{1}\right)} \\
& =\sqrt{1-2 \cos \left(2 \beta_{1}\right) \frac{g_{1}}{h_{1}} m+\frac{g_{1}^{2}}{h_{1}^{2}} m^{2}}+\frac{g_{1}}{h_{1}} m .
\end{aligned}
$$

To show this, we first obtain a polynomial equation for the $m$ that satisfies (64). Then we show that the polynomial equation for the $m$ satisfying (64) is exactly the same polynomial equation that returns the desired value of $m$ given in Theorem 1 .

From (64) we have

$$
\begin{gathered}
2 p\left(\sqrt{1-2 \cos \left(2 \beta_{1}\right) \frac{g_{1}}{h_{1}} m+\frac{g_{1}^{2}}{h_{1}^{2}} m^{2}}+\frac{g_{1}}{h_{1}} m-\cos \left(2 \beta_{1}\right)\right) \\
+q \sin ^{2}\left(2 \beta_{1}\right) \\
=2 \frac{g_{1}}{h_{1}} m\left(\sqrt{1-2 \cos \left(2 \beta_{1}\right) \frac{g_{1}}{h_{1}} m+\frac{g_{1}^{2}}{h_{1}^{2}} m^{2}}+\frac{g_{1}}{h_{1}} m\right. \\
\left.-\cos \left(2 \beta_{1}\right)\right) .
\end{gathered}
$$

Using (48) and noting that $0 \leq \bar{\theta}_{2} \leq \frac{\pi}{2}$, we have

$$
\begin{aligned}
\cos \left(2 \bar{\theta}_{2}\right) & =\frac{\cos \left(2 \beta_{2}\right)-\frac{g_{2}}{h_{2}} \frac{1}{m}}{\sqrt{1-2 \cos \left(2 \beta_{2}\right) \frac{g_{2}}{h_{2}} \frac{1}{m}+\frac{g_{2}^{2}}{h_{2}^{2}} \frac{1}{m^{2}}}} \\
& =\frac{m \cos \left(2 \beta_{2}\right)-\frac{g_{2}}{h_{2}}}{\sqrt{m^{2}-2 \cos \left(2 \beta_{2}\right) \frac{g_{2}}{h_{2}} m+\frac{g_{2}^{2}}{h_{2}^{2}}}} .
\end{aligned}
$$

Moreover, (51) leads to

$$
\begin{aligned}
\sqrt{1} & -2 \cos \left(2 \beta_{1}\right) \frac{g_{1}}{h_{1}} m+\frac{g_{1}^{2}}{h_{1}^{2}} m^{2} \\
= & \frac{h_{2}}{h_{1}} \sqrt{m^{2}-2 \cos \left(2 \beta_{2}\right) \frac{g_{2}}{h_{2}} m+\frac{g_{2}^{2}}{h_{2}^{2}}} \\
& +\frac{2}{h_{1}}\left(m\left(1+\frac{h_{2}}{2}+\frac{g_{1}}{2}\right)-\left(1+\frac{h_{1}}{2}+\frac{g_{2}}{2}\right)\right) .
\end{aligned}
$$

By applying (66), (67) in (65), some straightforward calculations yield the following polynomial equation for the $m$ satisfying (64):

$$
\begin{aligned}
& \left(\left(m-\frac{g_{2}}{h_{2}} \cos \left(2 \beta_{2}\right)\right)^{2}+\frac{g_{2}^{2}}{h_{2}^{2}} \sin ^{2}\left(2 \beta_{2}\right)\right) \\
& \quad \times\left(a m^{4}+b m^{3}+c m^{2}+d m+e\right)=0
\end{aligned}
$$

where the coefficients $a-e$ are exactly the same coefficients that represent the fourth degree polynomial equation defined in (32), and are given by (52)-(56).

Note that (68) has 6 roots, two of which are complex and clearly unacceptable ${ }^{4}$. Thus the root of interest for which (64) holds is among the roots of the fourth degree polynomial equation with the coefficients $a-e$. Since in obtaining (68) through (66), (67) we used the properties of the $m$ defined in Theorem 1, and since we reached the same polynomial equation as in Theorem 1, this clearly shows that the equality in (64) must hold. Consequently $\bar{\theta}_{1}=\widehat{\theta}_{1}$ or

$$
\bar{\theta}_{1}=\arg \max _{\theta_{1}} \mathcal{F}\left(\theta_{1}, \bar{\theta}_{2}\right) .
$$

Following the same steps as those above, we can show that

$$
\bar{\theta}_{2}=\arg \min _{\theta_{2}} \mathcal{F}\left(\bar{\theta}_{1}, \theta_{2}\right),
$$

which completes the proof of Theorem 2 .

\footnotetext{
${ }^{4}$ The complex roots are $\frac{g_{2}}{h_{2}}\left(\cos \left(2 \beta_{2}\right) \pm i \sin \left(2 \beta_{2}\right)\right)$.
} 


\section{REFERENCES}

[1] A. Wyner, “The wire-tap channel," Bell. Syst. Tech. J., vol. 54, no. 8, pp. 1355-1387, Jan. 1975.

[2] S. K. Leung-Yan-Cheong and M. E. Hellman, "The Gaussian wire-tap channel," IEEE Trans. Inf. Theory, vol. 24, pp. 451-456, Jul. 1978.

[3] F. Oggier and B. Hassibi, "The secrecy capacity of the MIMO wiretap channel," in Proc. IEEE Int. Symp. Inf. Theory, Toronto, ON, Canada, Jul. 2008, pp. 524-528.

[4] A. Khisti and G. Wornell, "Secure transmission with multiple antennas I: The MISOME wiretap channel," IEEE Trans. Inf. Theory, vol. 56, no. 7, pp. 3088-3104, 2010.

[5] A. Khisti and G. Wornell, "Secure transmission with multiple antennas II: The MIMOME wiretap channel," IEEE Trans. Inf. Theory, vol. 56, no. 11, pp. 5515-5532, 2010.

[6] T. Liu and S. Shamai (Shitz), "A note on secrecy capacity of the multiantenna wiretap channel," IEEE Trans. Inf. Theory, vol. 55, no. 6, pp. 2547-2553, 2009.

[7] R. Bustin, R. Liu, H. V. Poor, and S. Shamai (Shitz), "A MMSE approach to the secrecy capacity of the MIMO Gaussian wiretap channel," EURASIP J. Wireless Commun. Netw., vol. 2009, 2009, Article ID 370970, 8 pp.

[8] A. Mukherjee and A. L. Swindlehurst, "Optimal strategies for countering dual-threat jamming/eavesdropping-capable adversaries in MIMO channels," in Proc. IEEE MILCOM, San Jose, CA, Oct. 31-Nov. 3, 2010, pp. 1695-1700.

[9] S. A. A. Fakoorian and A. L. Swindlehurst, "Solutions for the MIMO Gaussian wiretap channel with a cooperative Jammer," IEEE Trans. Signal Process., vol. 59, no. 10, pp. 5013-5022, Oct. 2011.

[10] L. Dong, Z. Han, A. P. Petropulu, and H. V. Poor, "Improving wireless physical layer security via cooperating relays," IEEE Trans. Signal Process., vol. 58, no. 3, pp. 1875-1888, Mar. 2010.

[11] R. Zhang, L. Song, Z. Han, and B. Jiao, "Improve physical layer security in cooperative wireless network using distributed auction games," presented at the Proc. IEEE Infocom Workshop, Shanghai, China, 2011

[12] W. Saad, Z. Han, T. Başar, M. Debbah, and A. Hjørungnes, "Physical layer security: Coalitional games for distributed cooperation," presented at the Proc. 7th Int. Symp. Modeling Optim. Mobile, Ad Hoc, Wireless Netw. (WiOpt), Seoul, South Korea, Jun. 2009.

[13] Z. Han, N. Marina, M. Debbah, and A. Hjørungnes, "Physical layer security game: Interaction between source, eavesdropper, and friendly Jammer," EURASIP J. Wireless Commun. Netw., 2009 [Online]. Available: http://jwcn.eurasipjournals.com/content/2009/1/452907

[14] M. Yuksel, X. Liu, and E. Erkip, "A secure communication game with a relay helping the eavesdropper," IEEE Trans. Inf. Forensics Security, vol. 6 , no. 3, pp. 818-830, Sep. 2011.

[15] R. Liu, I. Maric, P. Spasojevic, and R. Yates, "Discrete memoryless interference and broadcast channels with confidential messages: Secrecy rate regions," IEEE Trans. Inf. Theory, vol. 54, no. 6, pp. 2493-2507, Jun. 2008

[16] E. A. Jorswieck and R. Mochaourab, "Secrecy rate region of MISO interference channel: Pareto boundary and non-cooperative games," presented at the Int. Workshop Smart Antennas (WSA), Berlin, Germany, Feb. 2009

[17] E. Toher, O. O. Koyluoglu, and H. El Gamal, "Secrecy games over the cognitive channel," in Proc. IEEE Int. Symp. Inf. Theory, Austin, TX, Aug. 2010, pp. 2637-2641.

[18] J. Xie and S. Ulukus, "Secrecy games on the one-sided interference channel," presented at the IEEE Int. Symp. Inf. Theory, Saint-Petersburg, Russia, Aug. 2011.

[19] S. A. A. Fakoorian and A. L. Swindlehurst, "MIMO interference channel with confidential messages: Achievable secrecy rates and beamforming design," IEEE Trans. Inf. Forensics Security, vol. 6, no. 3, pp. 640-649, Sep. 2011.

[20] M. J. Osborne and A. Rubinstein, A Course in Game Theory. Cambridge, MA: MIT Press, 1994.
[21] S. Shafiee and S. Ulukus, "Mutual information games in multiuser channels with correlated jamming," IEEE Trans. Inf. Theory, vol. 55, no. 10 , pp. $4598-4607$, Sep. 2009.

[22] R. Liu and H. Vincent Poor, "Secrecy capacity region of a multiple-antenna Gaussian broadcast channel with confidential messages," IEEE Trans. Inf. Theory, vol. 55, no. 3, pp. 1235-1249, Mar. 2009.

[23] E. A. Jorswieck, E. G. Larsson, and D. Danev, "Complete characterization of the Pareto boundary for the MISO interference channel," IEEE Trans. Signal Process., vol. 56, no. 10, pp. 5292-5296, Oct. 2008.

[24] M. Abramowitz and I. A. Stegun, Handbook of Mathematical Functions: With Formulas, Graphs, and Mathematical Tables. New York: Dover, 1972, pp. 17-18.

[25] R. A. Horn and C. R. Johnson, Matrix Analysis. Cambridge, U.K.: Cambridge Univ. Press, 1985.

[26] R. B. Myerson, Game Theory: Analysis of Conflict. Cambridge, MA: Harvard Univ. Press, 1997.

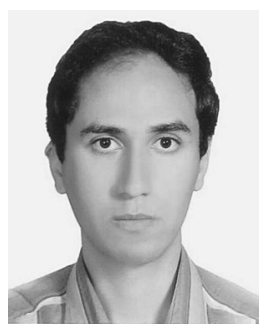

S. Ali A. Fakoorian (M'08) received the B.S. and M.S. degrees, both in electrical engineering, from Amirkabir University of Technology, Tehran, Iran, in 2002 and 2005, respectively.

$\mathrm{He}$ is currently working towards the Ph.D. degree at the Department of Electrical Engineering and Computer Science, University of California, Irvine. His research interests lie in multiple-user wireless networks, signal processing, and information theory.

Mr. Fakoorian is a student member of CPCC and the IEEE Communications and Signal Processing Societies.

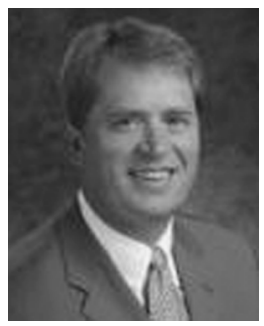

A. Lee Swindlehurst (F'04) received the B.S. (summa cum laude) and M.S. degrees in electrical engineering from Brigham Young University, Provo, UT, in 1985 and 1986, respectively, and the Ph.D. degree in electrical engineering from Stanford University, Stanford, CA, in 1991.

From 1986 to 1990 , he was with ESL, Inc. Sunnyvale, CA, where he was involved in the design of algorithms and architectures for several radar and sonar signal processing systems. He was on the faculty of the Department of Electrical and Computer Engineering at Brigham Young University from 1990 to 2007, where he was a Full Professor and served as Department Chair from 2003 to 2006. During 1996-1997, he held a joint appointment as a visiting scholar at both Uppsala University, Uppsala, Sweden, and at the Royal Institute of Technology, Stockholm, Sweden. From 2006 to 2007, he was on leave working as Vice-President of Research for ArrayComm LLC, San Jose, CA. He is currently a Professor of electrical engineering and computer science with the University of California at Irvine. His research interests include sensor array signal processing for radar and wireless communications, detection and estimation theory, and system identification, and he has over 200 publications in these areas.

Dr. Swindlehurst is a Past Secretary of the IEEE Signal Processing Society, past Editor-in-Chief of the IEEE JouRnal OF SELECTED TOPICS In Signal Processing, and past member of the Editorial Boards for the EURASIP Journal on Wireless Communications and Networking, IEEE Signal Processing Magazine, and the IEEE Transactions on Signal PROCESSING. He is a recipient of several paper awards: the 2000 IEEE W. R. G. Baker Prize Paper Award, the 2006 and 2010 IEEE Signal Processing Society Best Paper Award, and the 2006 IEEE Communications Society Stephen O. Rice Prize in the Field of Communication Theory, and he is coauthor of a paper that received the IEEE Signal Processing Society Young Author Best Paper Award in 2001. 